\title{
Constraints on the IMF and the brown dwarf population of the young cluster IC $348^{\star, \star \star}$
}

\author{
T. Preibisch ${ }^{1}$, T. Stanke ${ }^{1}$, and H. Zinnecker ${ }^{2}$ \\ 1 Max-Planck-Institut für Radioastronomie, Auf dem Hügel 69, 53121 Bonn, Germany \\ 2 Astrophysikalisches Institut Potsdam, An der Sternwarte 16, 14482 Potsdam, Germany
}

Received 28 January 2003 / Accepted 10 June 2003

\begin{abstract}
We use a deep near-infrared census of the young stellar cluster IC 348 to construct and analyze its luminosity function. Our mosaic image of IC 348 covers the full extent of the cluster with a completeness limit of $J \sim 19.5$ and is therefore sensitive for $2 \mathrm{Myr}$ old cluster members with masses as low as $M \geq 0.005 M_{\odot}$ for the mean extinction of the known cluster members $\left(A_{V} \sim 3.5 \mathrm{mag}\right)$. By using information on stellar ages, extinctions, and the binary population in IC 348 from several recent studies, we can derive statistical constraints on the stellar and sub-stellar mass function of the cluster by modeling the observed luminosity function. We find that the stellar part of the mass function in IC 348 is well described by the galactic field star IMF. While several brown dwarfs have recently been identified in IC 348, our data show that the cluster harbors only a relatively small population of sub-stellar objects. We find that brown dwarfs in the mass range $0.02-0.075 M_{\odot}$ constitute at most $\sim 10 \%$ of the total cluster population, in contrast to recent results suggesting much larger brown dwarf populations in other young clusters and also the galactic field. Our results suggest that IC 348 has $\sim 2 \times$ fewer brown dwarfs than the Orion Trapezium cluster. A similar brown dwarf "deficit" was recently found in the Taurus star forming region. We speculate about the possible causes for this result, including the presence or absence of nearby massive stars and their influence on the formation of low-mass young stellar objects.
\end{abstract}

Key words. stars: pre-main sequence - stars: low-mass, brown dwarfs - stars: luminosity function, mass function open clusters and associations: general

\section{Introduction}

The initial mass function (IMF) is of utmost importance for any theory of star formation. While the theoretical expectation is that the IMF should vary systematically with the star formation environment, no really convincing evidence for variations in the stellar part of the IMF has yet been found (for recent reviews see Kroupa 2001, 2002; Scalo 1998). The effects of environment may be clearest at the low-mass end of the IMF, as one might imagine that the least massive protostars are most strongly affected by external effects. The sub-stellar part $\left(<0.075 M_{\odot}\right)$ of the IMF, which is not as well characterized as the stellar mass function, is therefore a good point to look for variations in different environments.

After the first detection of BDs (see Rebolo et al. 1995; Oppenheimer et al. 1995) numerous sub-stellar objects have

Send offprint requests to: T. Preibisch,

e-mail: preib@mpifr-bonn.mpg.de

* Based on observations obtained at the German-Spanish Astronomical Centre, Calar Alto, operated by the Max-PlanckInstiute for Astronomy, Heidelberg, jointly with the Spanish National Commission for Astronomy.

$\star \star$ Table 1 is only available in electronic form at http://www. edpsciences.org been found in (young) clusters and also in the galactic field (for an overview see Oppenheimer et al. 2000 or the proceedings of the IAU Symp. 211 on Brown Dwarfs; Martin 2003). During the last couple of years, important steps in the characterization of the sub-stellar mass function have been made (e.g. Reid 1999). In a summary of recent results Chabrier (2002) concluded that the total number of BDs (in the mass range $0.001-0.07 M_{\odot}$ ) in the Galactic disk is similar to the number of stars.

IC 348 is a very young cluster located in the Perseus molecular cloud complex at a distance of about 310 pc (cf. Herbig 1998). For a long time, the cluster was believed to consist of just a dozen T Tauri members (Herbig 1954, Harris et al. 1954), but recent sensitive optical (Herbig 1998), nearinfrared (Lada \& Lada 1995; Luhman et al. 1998), and Xray observations (Preibisch et al. 1996; Preibisch \& Zinnecker 2001, 2002) have shown that IC 348 contains more than 100 stars. Luhman et al. (1998) showed that the mass spectrum in IC 348 extends well into the sub-stellar regime. In a deep near-infrared spectroscopic survey of faint objects in IC 348, which later was extended by Luhman (1999), at least 8 objects could be identified which appear to have masses below $0.075 M_{\odot}$, i.e. the hydrogen-burning mass-limit. 
Najita et al. (2000) [N00 hereafter] identified further browndwarf candidates by deep HST/NICMOS narrow-band imaging, using the $1.9 \mu \mathrm{m}$ water absorption band strength as an indicator of spectral type. Their observations covered the $5^{\prime} \times 5^{\prime}$ core of IC 348, are complete down to masses of $\sim 0.02 M_{\odot}$, and revealed 20-30 substellar candidates. Liu (2002) performed $K$ band spectroscopy for some of the BD candidates in IC 348 at the Subaru $8 \mathrm{~m}$ Telescope and found that for the faintest objects only marginally useful spectra can be obtained. This demonstrates that a spectroscopic identification of the full population of BDs, down to the deuterium burning mass limit at $0.015 M_{\odot}$ and across the whole cluster area, would require a large amount of observing time. The existing samples of spectroscopically identified BDs are necessarily subject to the problems of small number statistics.

On the other hand, near-infrared photometric observations of a compact cluster like IC 348 can rather easily go deep enough to detect all cluster members down to masses below $\sim 0.01 M_{\odot}$. It is thus relatively easy to determine the full cluster luminosity function. Modeling of the observed luminosity function (cf. Zinnecker et al. 1993) then allows one to obtain statistical constraints on the mass function. An early attempt to gain information about the low-mass and sub-stellar IMF on the basis of an observed luminosity function was presented by McCaughrean et al. (1995) for the case of the Trapezium cluster.

In general, the knowledge of the luminosity function is not sufficient to draw strong conclusions on the underlying mass function, because the individual stellar magnitudes are not only a function of the stellar mass, but also strongly depend on the stellar age and the extinction. If, however, the distribution of stellar ages and extinctions is known as a piece of independent information, this ambiguity is largely removed and the luminosity function basically depends only on the mass function. Although it is clear that any luminosity function modeling procedure can give only statistical constraints on the mass function, i.e. yields less information than a (spectroscopic) oneby-one identification of cluster members, it allows nevertheless important insights with a relatively small observational effort.

While many similar studies of cluster luminosity functions (e.g. Zinnecker et al. 1993; Lada et al. 1998) have been performed in the $K$-band $(2.2 \mu \mathrm{m})$, we have chosen the $J$-band $(1.2 \mu \mathrm{m})$ for our study of IC 348 . Our choice was motivated by the fact that the $J$-band flux seems to be a better tracer of the stellar bolometric luminosity (cf. Kenyon \& Hartmann 1995). This is even more important as we are dealing with very young stars, for which the $K$-band flux is often strongly affected by excess emission due to hot circumstellar material, most pronounced in the case of the classical T Tauri stars. In the $J$-band, this excess emission is much reduced. The $K$-band has the advantage of being less sensitive to extinction than the $J$-band $\left(A_{K} \approx 0.11 \times A_{V} ; A_{J} \approx 0.28 \times A_{V} ;\right.$ cf. Rieke \& Lebofsky 1985$)$, but this is not very relevant for our particular study, since the extinction of the stars in IC 348 is not very large $\left(A_{J} \lesssim 3 \mathrm{mag}\right.$; see below).

For the distance of IC 348 we adopt here a value of $310 \mathrm{pc}$ (see $\mathrm{H} 98$ for a detailed justification of this value). We note that IC 348 is associated to the Per OB2 association, and the distance we use is in very good agreement with the recent determination of Per OB2 distance $(318 \pm 27$ pc; de Zeeuw et al. 1999) based on a detailed analysis of the Hipparcos data. Also, the recent detection of $\delta$ Scuti-like pulsations in one of the F-type stars in IC 348 by Ripepi et al. (2002) allowed an independent distance determination that is in full agreement with our assumption of $310 \mathrm{pc}$ and rules out earlier suggested values of only about $250 \mathrm{pc}$ (e.g. Cernis 1993).

\section{Deep J-band imaging of IC 348}

\subsection{Observations and data reduction}

The observations discussed in this paper were obtained during the night of 2nd Dec. 2001 using the Omega Prime wide-field near-infrared camera (Bizenberger et al. 1998) on the Calar Alto $3.5 \mathrm{~m}$ telescope in service observing mode. The camera uses a $1024 \times 1024$ pixel $\mathrm{HgCdTe}$ array and provides a $6.7^{\prime} \times 6.7^{\prime}$ field-of-view at a pixel scale of $0.4^{\prime \prime}$. The images were taken through a standard $J$-band filter $(1.13-1.42 \mu \mathrm{m})$. We obtained a $3 \times 3$ mosaic image covering an $18^{\prime} \times 18^{\prime}$ area around IC 348. Furthermore, we also obtained two background images about $30^{\prime}$ east and west of IC 348 , each covering a $6^{\prime} \times 12^{\prime}$ area. The stepsize between the mosaic positions was chosen such that a significant overlap between the frames allowed accurate registration of the images relative to each other. At each mosaic position, a sequence of 15 images with individual exposure time of $20 \mathrm{~s}$ was repeated in 5 dither positions with offsets of about $20^{\prime \prime}$. The final per pixel integration time is thus $5 \times 15 \times 20 \mathrm{~s}=1500 \mathrm{~s}(25 \mathrm{~min})$. The observing conditions were photometric during the whole night, and the mean seeing was $1.3^{\prime \prime}$.

The data reduction followed standard infrared procedures. For each individual exposure, sky frames were constructed by median averaging (to remove stellar images) of adjacent (in time and on the sky) exposures. These were then subtracted from the frames, thus also removing the bias level. Bad pixels were masked and excluded from further processing. The frames were then divided by a normalized flat-field, which was constructed of exposures of the dome, once with a flat-field lamp on, then off. Finally, the images were registered and median averaged to reject cosmic ray events. As the edges of the final mosaic constructed from the dithered exposures have not the full integration time, only that part of the mosaic with the full 25 minutes integration time was used for further analysis. This final image measures $18.6^{\prime} \times 18.4^{\prime}$, giving a field of view of $\sim 340$ square-arcminutes. The center of our final image is at the position RA $=3^{\mathrm{h}} 44^{\mathrm{m}} 30^{\mathrm{s}}$, Dec $=32^{\circ} 08^{\prime} 00^{\prime \prime}(\mathrm{J} 2000)$. The sky fields both measured about 72 square-arcmin. The UKIRT faint infrared standard star FS 113 was observed for photometric calibration.

\subsection{Photometry}

The MIDAS INVENTORY package was used for the source detection, and the resulting source list carefully checked for apparent misidentifications such as multiple detections of strongly saturated sources, wrong source detections in 
diffraction spikes of bright sources, extended, nebulous features, and the $\mathrm{H}_{2}$ features associated with the outflows in the HH211 region. 1991 sources were thus found on the cluster mosaic, and 632 and 662 sources were found in the comparison fields. Aperture photometry was then done using the DAOPHOT package, dealing separately with the subfields of the cluster mosaic and the comparison fields. This was done in order to correct the photometry for the actual atmospheric extinction and to allow for apertures varying as the actual seeing. Thereafter the photometry was carefully checked for possible cloud contamination by first comparing brightness measurements for the individual exposures taken on each subfield, and then comparing brightness measurements for stars in the overlap region between the subfields. We did not find any evidence for intervening clouds, with the brightness measurements scattering only little (typically 0.02 mag for reasonably bright stars) around the mean value. We thus regard the data to be taken under photometric conditions.

The photometry was also compared with the 2MASS data. Again, there was a very good correspondence between the two data sets, although there is a small offset between the two (2MASS yields about 0.05-0.1 mag fainter stars; note however that our measurements do not show any significant offset from the photometry obtained by Lada \& Lada 1995). This indicates that the uncertainty of the absolute calibration of our data is 0.1 mag or less. Furthermore, the comparison between 2MASS and our photometry suggests that our data suffer from saturation for stars brighter than about $J \sim 12$. Thus for stars brighter than $J=12.5$ the photometry from 2MASS was used instead of our own measurements. The comparison of brightness measurements obtained from the individual exposures suggests that measurement errors are less than 0.1 mag for stars as faint as $J \sim 18-18.5$.

The limiting magnitude of the exposures was found to be at $J \sim 21$. The number of stars per magnitude closely follows a power-law. The completeness limit of our images can be approximated as the magnitude at which the distribution starts to deviate from a power-law relation, which we find at $J_{\text {compl. }}=19.5$. A similar result was also found by adding artificial stars to the images and applying the same detection techniques as described above. Comparing this with the expected magnitudes of 2 Myr old BDs (B98; Baraffe et al. 2003) at the distance of IC 348 , we conclude that our data are complete to all BDs with masses of $0.005 M_{\odot}$ or more for the typical extinction of the known cluster members $\left(A_{V} \sim 3.5 \mathrm{mag}\right)$ and to objects with masses of $0.02 M_{\odot}$ or more for extinctions of up to $A_{V}=11 \mathrm{mag}$.

The BDs identified by Luhman (1999) are clearly visible in our data and are marked in Fig. 1. A table containing the positions and magnitudes of all stars detected in our image is given in Table 1 (available in electronic form only).

\section{Determination of the cluster luminosity function}

\subsection{Definition of the cluster area}

The first step in the determination of the $J$-band luminosity function (JLF) of IC 348 is the definition of the cluster area.
Table 1. $J$-band magnitudes of the sources detected in our IC 348 image. The source names contain the $\mathrm{J} 2000$ coorinates. The complete version of this table is in electronic form. The printed edition contains only a sample.

\begin{tabular}{lc}
\hline \hline IC348-J & $\begin{array}{c}J \\
{[\mathrm{mag}]}\end{array}$ \\
\hline \hline $034350.6+321059$ & 18.69 \\
$034350.6+321231$ & 19.46 \\
$034350.7+321306$ & 14.20 \\
$034350.7+321125$ & 19.98 \\
$034350.8+321433$ & 19.65 \\
$\ldots$ & $\ldots$ \\
$\ldots$ & $\ldots$ \\
$\ldots$ & $\ldots$ \\
\hline
\end{tabular}

The spatial distribution of objects in our mosaic image shows an excess in the surface density of objects up to radii of $5^{\prime}-6^{\prime}$ from the cluster center; this is in good agreement with the results of H98 and of Lada \& Lada (1995). We therefore use a cluster radius of $6^{\prime}$ for the following analysis.

\subsection{Magnitude distributions in the cluster and background fields}

We constructed histograms of the $J$-band magnitudes of all point sources located in the cluster area and the background areas; these histograms are shown in Fig. 2. The cluster area contains 762 objects, the two background areas contain 1294 objects.

The number of objects per magnitude interval in the background fields closely follows a power-law, as expected for a uniformly distributed background population. The cluster field histogram also shows a power-law like increase in the number of objects for faint magnitudes $(J \gtrsim 16)$, but in the magnitude range $J \sim 10-16$ the distribution shows a strong bump, which represents the cluster population.

\subsection{Background subtraction}

In order to properly correct the cluster area histogram for the background population in the cluster field, we need a model for the distribution of background objects in the cluster area. To construct this background model, we scaled the background magnitude histogram according to the areas covered by our cluster field and the background fields. Furthermore, we also have to take into account the difference in interstellar extinction along the line-of-sight (LOS) to the background fields as compared to the LOS in the direction of IC 348. What we need to determine is the amount of additional extinction along the IC 348 LOS in excess of the background field LOS extinction.

IC 348 is located at the eastern edge of the Perseus molecular cloud complex. A dense cloud core about $10^{\prime}$ to the southwest of the cluster center contains several deeply embedded infrared sources with extinctions exceeding $\sim 20 \mathrm{mag}$ in $A_{V}$ as well as the very young molecular hydrogen jet $\mathrm{HH} 211$ (McCaughrean et al. 1994) and the IC 348 MMS outflow 


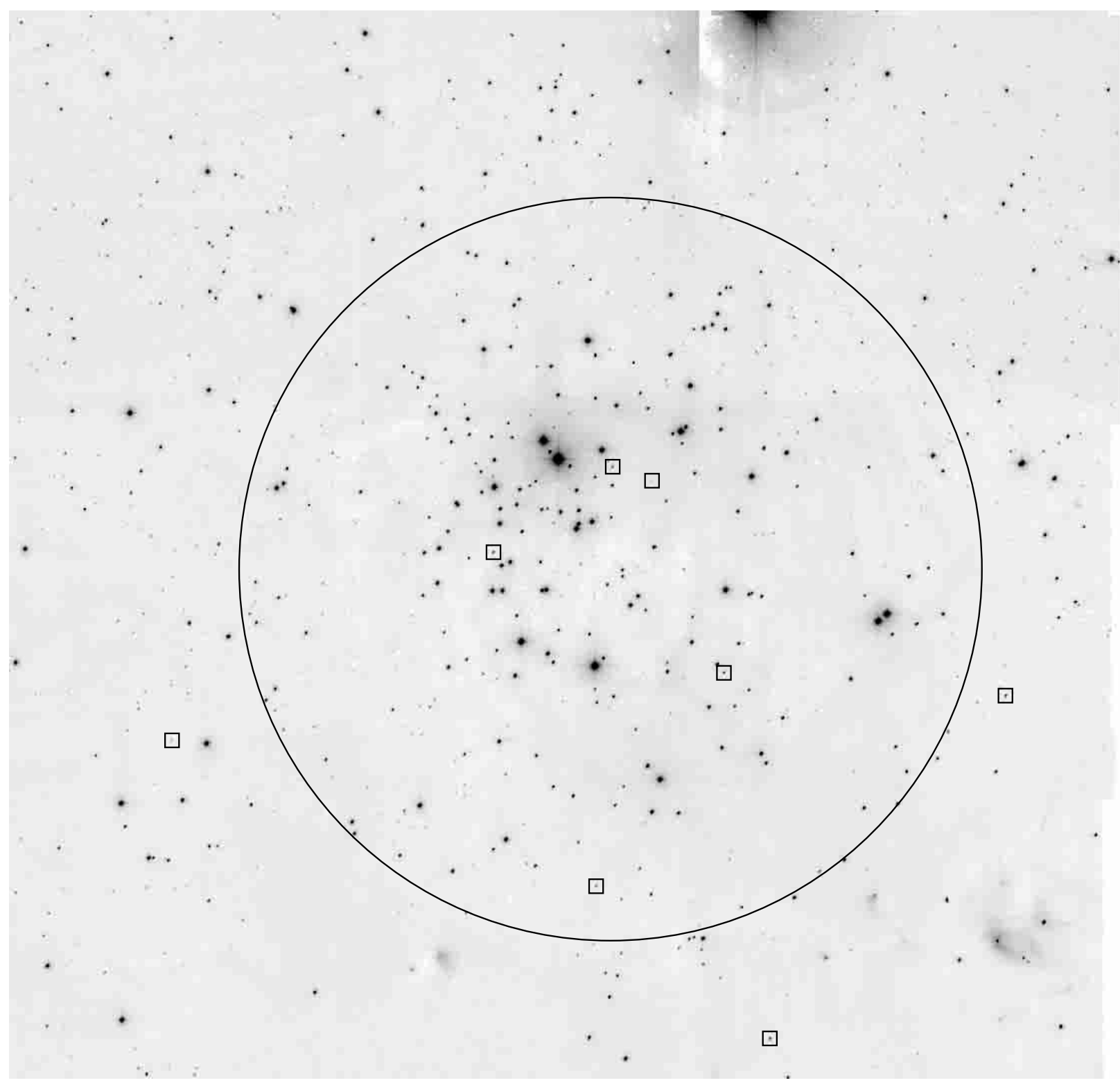

Fig. 1. Our $J$-band mosaic image of IC 348 , showing a $18.6^{\prime} \times 18.4^{\prime}$ field with a square-root intensity scale. The eight BDs (objects with spectral types $\geq$ M 6.5) identified by Luhman (1999) are marked by boxes. The large circle marks the cluster area.

(Eislöffel et al. 2003). In the central cluster area, however, the cloud extinction is much lower. Cernis (1993) found that the whole region is covered by a foreground layer of diffuse extinction with $A_{V} \sim 0.7 \mathrm{mag}$, in good agreement with the results obtained earlier by Cernicharo et al. (1985). Both studies also found a second layer of absorbing material, the dark clouds in the Perseus complex, producing extinctions of several magnitudes.

A quantitative estimate of the extinction caused by the IC 348 cloud can be based on the extinctions derived for some 50 background stars in the central cluster area by N00, which show a broad distribution from $A_{V} \sim 1$ mag up to $A_{V} \sim 10-12$ mag.

For the extinction in the direction of our two background fields, we can use the reddening maps of Schlegel et al. (1998), which suggest extinctions between $A_{V} \lesssim 2$ mag and
$A_{V} \sim 6.5$ mag. Given these results, we have to add $\sim 4$ mag of extinction to the background data to correct for this difference. This corresponds to $\Delta A_{J}=1.1 \mathrm{mag}$.

\subsection{The cluster JLF}

The cluster JLF can now be determined by subtracting the reddened background histogram from the cluster field histogram. The resulting cluster JLF can be seen in Fig. 3. The most important features of the cluster JLF are a pronounced peak at $J \sim 13.5$ and the strong drop in the range $J \sim 14-16$. The histogram shows a number of objects in the $J \sim 17.5-19.5$ range, but this is not significant and can be fully explained as a statistical fluctuation of the background subtraction. In the following plots we therefore show these insignificant histogram bins only by thin dotted lines. 


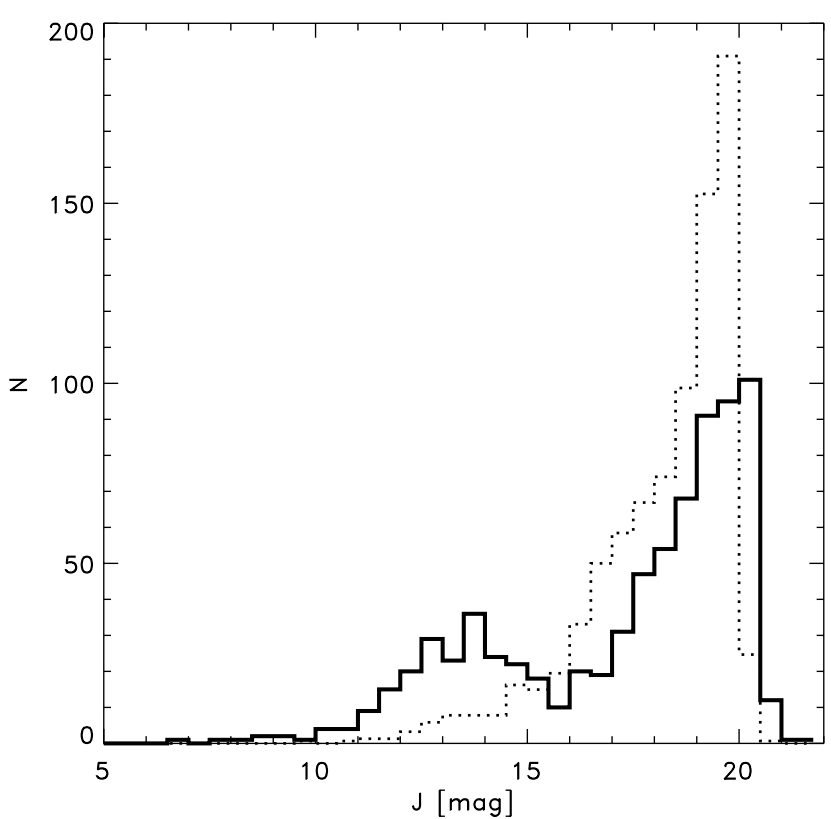

Fig. 2. Histogram of $J$-band magnitudes for the central $6^{\prime}$ radius area in our IC 348 cluster image (thick solid line) compared to the histogram for the background fields (dotted line, scaled to the same area as the cluster region).

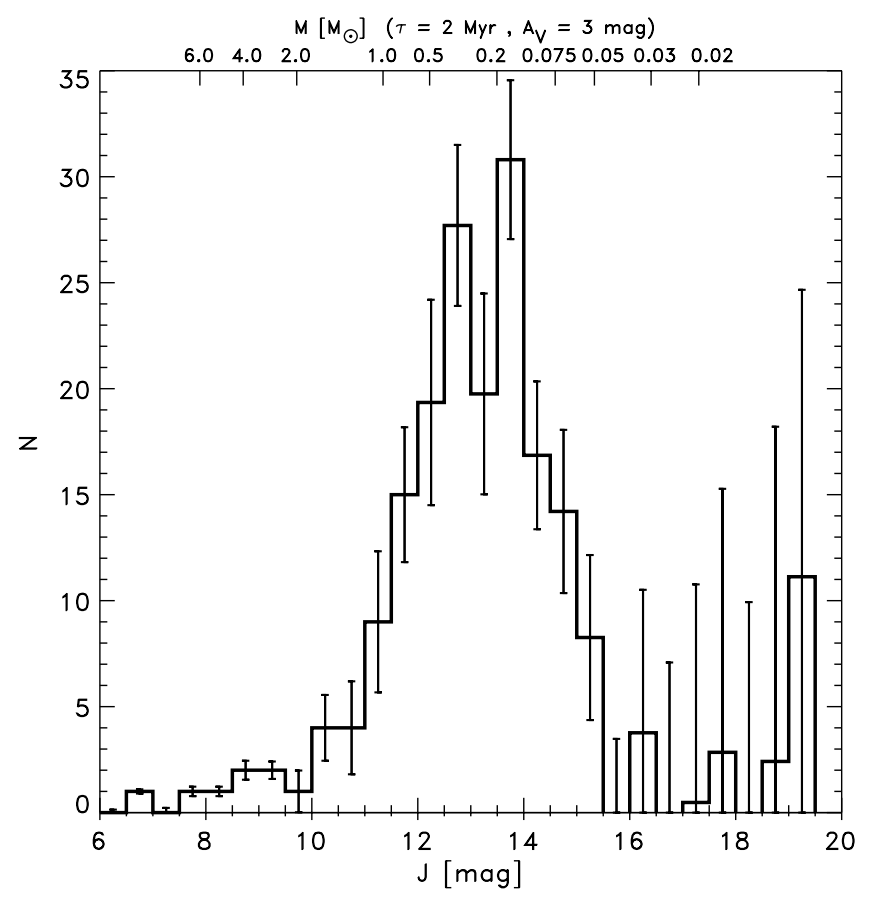

Fig. 3. JLF of IC 348, computed by subtracting the background model histogram from the cluster area histogram. The error bars represent the uncertainties caused by the photometric errors $(\sigma \sim 0.05 \mathrm{mag})$ and the Poisson statistical uncertainties of the background subtraction.

\section{JLF modeling procedure}

We performed detailed simulations to model the observed JLF of IC 348. The general outline of our Monte-Carlo simulation procedure is as follows: In the first step, we randomly draw 30000 stellar masses from a distribution specified by the input IMF. For each simulated star we draw a value for the stellar age from the age distribution described below. Then the $J$-band magnitude of each simulated star is determined according to its mass and age using pre-main sequence (PMS) evolutionary models. Finally, each star is extincted by randomly drawing a value for $A_{V}$ from the distribution described below.

\subsection{Mass function}

In this paper we will mainly use power-law functions to describe the mass function:

$\Psi(M)=\frac{\mathrm{d} N}{\mathrm{~d} M}=c M^{\alpha} \quad$ for $\quad M_{\min } \leq M \leq M_{\max }$,

where $\Psi(M)$ gives the number of stars per unit mass. We note that some authors prefer to use the number of stars per unit $\log$ mass, $\xi(M)=\frac{\mathrm{d} N}{\mathrm{~d} \log M}$, and that $\Psi(M) \propto M^{\alpha}$ corresponds to $\xi(M) \propto M^{\alpha+1}$.

\subsection{Stellar ages}

The ages derived for individual stars by H98 and L98 by comparison with PMS model isochrones range from less than $1 \mathrm{Myr}$ to about $10 \mathrm{Myr}$, with the majority of the objects having ages of a few Myr (a mean age of 1.3 Myr was derived by H98). It is important to note that the ages determined by comparison with PMS model isochrones, which we will denote "isochronal ages", are not identical to the true ages of the objects. The apparent age spread derived from individual isochronal ages is always larger than the true age spread, because any measurement errors and the intrinsic variability of the young stars will increase the apparent spread in the isochronal ages. Another important factor are unresolved binary systems, which will lead to systematically overestimated luminosities which in turn transform into too young isochronal ages. In summary, the true mean age of a stellar population will be somewhat larger than the mean of the isochronal ages, and the true age spread will always be smaller than the scatter of the isochronal ages. A detailed analysis of these effects can be found in Preibisch \& Zinnecker (1999).

We consider both effects in our simulations as follows. In our Monte-Carlo programs we draw ages from a Gaussian distribution with $\langle\log (\tau)\rangle=6.3$ and $\sigma_{\log (\tau)}=0.1$. If an age of less than $0.7 \mathrm{Myr}$ or larger than $7 \mathrm{Myr}$ is drawn, the drawing is repeated. The mean value of the resulting age distribution is $2 \mathrm{Myr}$; this is consistent with the mean "isochronal age" of $\sim 1.3 \mathrm{Myr}$ if a binary fraction of $\sim 50 \%$ is assumed. The width of the simulated age distribution is also roughly consistent with the apparent spread of "isochronal ages", if the effect of measurement errors is considered.

\subsection{Extinction of the cluster members}

The extinctions of the stars in IC 348 derived by H98 and L98 range from $A_{V} \sim 1 \mathrm{mag}$ to $A_{V} \lesssim 10 \mathrm{mag}$, with a mean of $A_{V} \sim 3.5 \mathrm{mag}$. The NICMOS study of N00 revealed several stars with somewhat higher extinctions. In our simulations, we randomly draw values for the extinction from a halfGaussian distribution (i.e. only positive numbers are used) with 
a width $\sigma\left(A_{V}\right)=4 \mathrm{mag}$ and increase every extinction value by $A_{V}=0.7 \mathrm{mag}$ (to account for the diffuse foreground extinction mentioned above). The corresponding values range from $0.7 \mathrm{mag}$ to $\approx 20 \mathrm{mag}$ with a mean of $3.9 \mathrm{mag}$. The resulting distribution is very similar to the empiric distribution found by N00 and also consistent with the results of H98 and L98.

\subsection{PMS evolutionary models}

We use the recent version of the Baraffe et al. (1998) models (see also Chabrier \& Baraffe 2000) which have been found to be very well consistent with observational constraints (cf. Luhman 1999; White et al. 1999). These models have the additional advantage that they give not only the stellar luminosity and effective temperature as a function of mass and age, but also the magnitudes in observational passbands. So there is no need to transform luminosities and effective temperatures to magnitudes and the uncertainties in the calibration of these transformations can be avoided.

The Baraffe et al. models start at an age of $1 \mathrm{Myr}$ and cover masses from $0.02 M_{\odot}$ up to $1.2 M_{\odot}$. Since IC 348 contains also some stars with higher masses (the most massive member is the $\mathrm{B} 5$ star $\mathrm{BD}+31^{\circ} 643$ with $M \sim 6 M_{\odot}$ ), we used the Palla \& Stahler (1999) models for masses above $1.0 M_{\odot}$. In the range of ages we need here, these two sets of models agree reasonably well at $1 M_{\odot}$. In order to compute the $J$-band magnitudes of the stars with $M \geq 1.0 M_{\odot}$, we use the intrinsic colors and bolometric corrections as a function of effective temperature from the tabulation in Kenyon \& Hartmann (1995) and Leggett et al. (1996).

\subsection{Unresolved binaries}

It is well known that many, perhaps most stars are members of multiple systems. About $50 \%$ of the field stars in the solar neighborhood are binaries or higher order multiple systems (cf. Duquennoy \& Mayor 1991; Fischer \& Marcy 1992). A recent study of the binary population in IC 348 has been performed by Duchene et al. (1999), who conducted a nearinfrared adaptive optics survey of 66 members of IC 348 and found 12 binary systems in the separation range $0.1^{\prime \prime}-8^{\prime \prime}$. They concluded that the binary fraction in IC 348 is consistent with that in the solar neighborhood, i.e. that IC 348 displays no evidence for an overabundance of multiple systems. We therefore assume that $50 \%$ of the stars in IC 348 are binaries, most of which would be unresolved in our image.

The effects of unresolved binary components are modeled in the following way: To each primary star we randomly add a companion with a probability equal to the assumed binary fraction (50\%). The companion mass is drawn from the same IMF as the primary mass, but with the restriction that it must be smaller than the primary mass. For these simulated binary systems we use the combined system magnitude, containing the flux from the primary and the companion, instead of the primary magnitude.

\subsection{Fit quality}

In order to evaluate how good (or bad) the different model IMFs reproduce the observed cluster JLF, we will compare below the histograms of the cluster JLF to the corresponding histograms computed from the model IMFs. The normalization of the model IMF histograms was chosen so that it reproduces the number of objects in the $J=10-14$ mag range.

Since we also would like to decide whether a certain model provides a statistically acceptable description of the data and to compare the success of different models in reproducing the observed JLF, we need a quantitative estimate of the "fit quality". While in many similar studies a $\chi^{2}$ test is used for this purpose, we note that this test is not completely suitable here: it provides a correct estimate for the probability that a specific model is consistent with the observed data only if the errors are normally distributed, which is not the case here. We therefore decided to use a Kolmogorov-Smirnov two-sample test (KS test), which is a non-parametric test and thus yields correct probabilities without conditions for the distribution of the errors. A KS test between the observed cluster JLF (in which we treat the histogram points at $J>17$ as statistical fluctuations, i.e. assume them to be zero) and the model JLF gives the probability that the deviations between the model and the data are purely caused by statistical fluctuations. For the interpretation of the resulting probabilities we note that a model with $P_{\mathrm{KS}} \gtrsim 0.5$ provides a reasonably good description of the data, while a probability of, e.g. $P_{\mathrm{KS}}=0.05$ shows that the model can be rejected at the $95 \%$ confidence level and can be considered to be invalid.

In Table 2 we summarize all relevant data for each of the different IMF models considered in this paper: the analytic formula for the mass function $\mathrm{d} N / \mathrm{d} M$, the "fraction of BDs" which we define as the fraction of objects in the mass range $0.02-0.075 M_{\odot}$ among all objects computed by integration of the mass function, and finally the KS test probability. A comparison of most of the mass functions considered in this paper is shown in Fig. 8.

\subsection{Uncertainties of the modeling}

It is clear that the reliability of the modeling results depends on the validity of the assumption on ages, extinctions, binary fractions and the correctness of the employed PMS models. As discussed above, due to the numerous studies of IC 348 , we can use relatively well founded and thus reliable assumptions for these factors. In order to see to what extent our results might depend on the use of one specific PMS model, we have also performed some tests with different sets of models. For this we firstly employed the D'Antona \& Mazzitelli (1997) models, and secondly also a combination of the Palla \& Stahler (1999) models for masses $0.1 \leq M / M_{\odot} \leq 6$ with the Burrows et al. (1997) models for masses $0.02<M / M_{\odot} \leq 0.1$. We found that the use of these models instead of the B98 models generally causes only slight differences in the shapes of the simulated luminosity functions. The IMF power-law slopes $\alpha$ inferred from the fits to the observed luminosity function with the different models do generally not differ by more than $\sim 0.1$. 


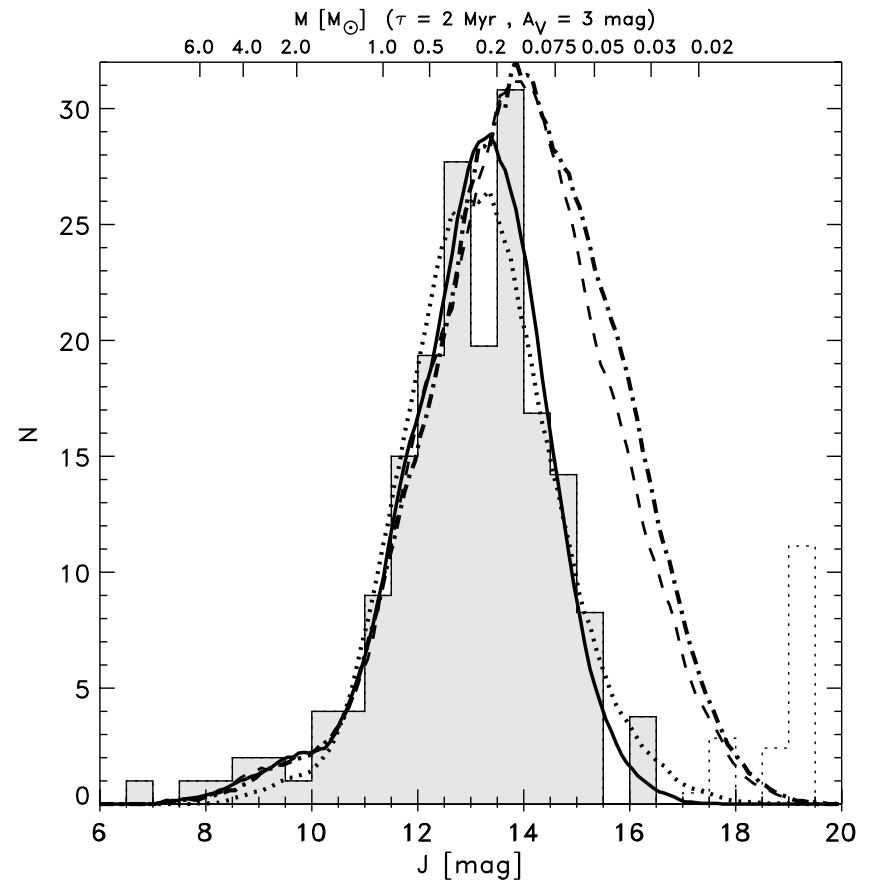

Fig. 4. The IC 348 JLF (histogram) compared to a model based on the Kroupa (2002) field IMF (dashed line), the stellar part of the Kroupa (2002) field IMF (solid line), the Chabrier (2002) log-norm IMF (dashed-dotted line), and our log-norm IMF fit (dotted line).

Also, the derived sizes of the substellar population are quite similar for the different models. The differences in the IMF determinations with different PMS models are rather small because the luminosities predicted by the different models for PMS objects with ages of a few Myr agree rather well (for a more detailed discussion of these differences see Muench et al. 2002). Therefore, none of our conclusions drawn in this paper depends significantly on the choice of a specific PMS model.

\section{JLF modeling}

\subsection{Comparison with the galactic field IMF}

We will start our modeling with recent representations of the galactic field IMF. We first consider the following parameterization for the average galactic field IMF in the solar neighborhood given by Kroupa (2002):

$\frac{\mathrm{d} N}{\mathrm{~d} M} \propto\left\{\begin{array}{llr}M^{-0.3} & \text { for } & 0.02<M / M_{\odot}<0.08 \\ M^{-1.3} & \text { for } 0.08<M / M_{\odot}<0.5 \\ M^{-2.3} & \text { for } 0.5<M / M_{\odot}<100\end{array}\right.$

or, in the shorter notation we will use in the following text, $\alpha[0.02-0.08]=-0.3, \alpha[0.08-0.5]=-1.3, \alpha[0.5-100]=$ -2.3 . The comparison of the cluster JLF with the model based on the Kroupa (2002) IMF is shown in Fig. 4. This mass function obviously predicts much more objects at magnitudes $J>14$ than observed in IC 348. The disagreement between model and data is highly significant, what is clearly confirmed by the very small KS test probability of $P_{\mathrm{KS}}=4.12 \times 10^{-10}$. This implies that IC 348 contains a significantly smaller number of BDs than expected from the Kroupa (2002) IMF. As the

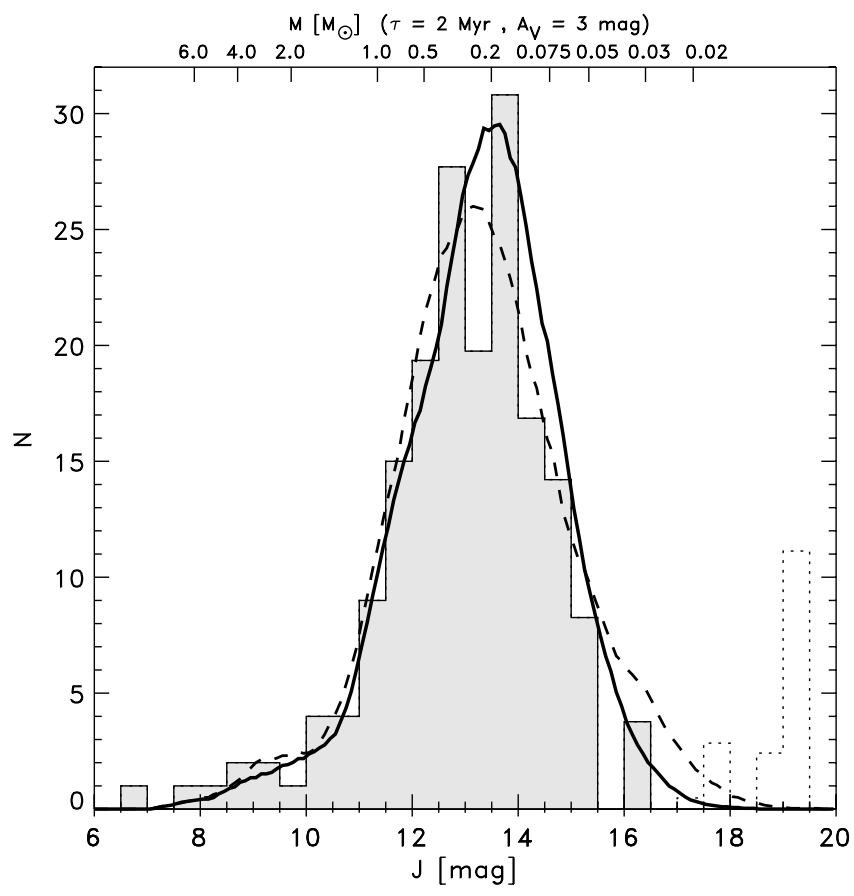

Fig. 5. The IC 348 JLF (histogram) compared to the two marginally acceptable Kroupa IMF like models with maximum BD population. The solid line shows the model with the power-law slope $\alpha=+2.5$ in the sub-stellar part (model "Fit I"), the dashed line shows the model "Fit II", in which the change of the power-law slope occurs at $0.22 M_{\odot}$.

"fraction of BDs" found by integrating the model IMF is $30 \%$ (see Table 2, the actual fraction of BDs in IC 348 must be much smaller than this value.

Next we consider a purely stellar IMF, which is identical to the Kroupa (2002) IMF for masses above $0.08 M_{\odot}$ and contains no BDs. The corresponding model JLF is compared to our observed JLF in Fig. 4 and can be seen to provide a rather good description of the observed JLF ( $\left.P_{\mathrm{KS}}=0.975\right)$. This good agreement also shows that our above assumptions about the cluster properties are reasonable and provide a consistent description of the data. As closer look reveals that the purely stellar IMF predicts slightly too few objects for $J \geq 15$; this suggests the presence of a small BD population in IC 348 (the existence of which was of course already proven by the spectroscopic identification of a few BDs by N00 and L00).

Chabrier (2002) included the results of recent near-infrared surveys (DENIS, 2MASS, SLOAN DSS) in his determination of the stellar and sub-stellar mass function in the Galactic disk. He proposed several possible representations of the IMF, including a log-normal function. This model assumes a Gaussian distribution in $\log M$ with a peak at $0.1 M_{\odot}$ and a width of $\sigma(\log M)=0.627$ (see Table 1 for details). It yields a model JLF which is very similar to that for the Kroupa (2002) IMF and is not consistent with our JLF for IC 348.

We investigated in which way the parameters of the lognormal distribution have to be changed in order to find a good agreement between the observed cluster JLF and the model. The best fit (i.e. the highest $P_{\mathrm{KS}}$ value) is found for a model in which the mass function is given by a Gaussian distribution in $\log M$ with a peak at $0.25 M_{\odot}$ and a width of $\sigma(\log M)=0.4$. 


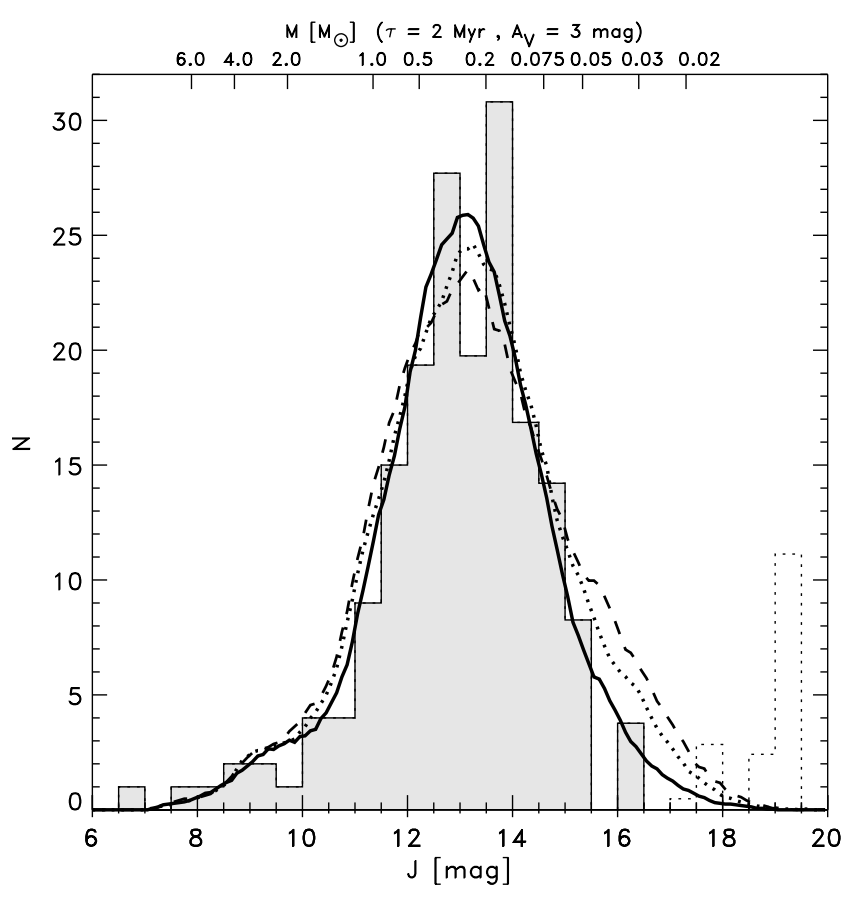

Fig. 6. The IC 348 JLF (histogram) compared to the IMF models for IC 348 derived by Lada et al. (1998); the model L1 is shown by the dashed line, model L2 by the dotted line. The solid line shows the model based on the IMF suggested by Luhman et al. (2000) for IC 348 .

This model has a BD fraction of $9 \%$, yields $P_{\mathrm{KS}}=0.777$ and is also shown in Fig. 4.

\subsection{Constraining the sub-stellar IMF of IC 348}

We now want to determine an upper limit to the size the of BD population in IC 348 by considering the following question: How must the Kroupa IMF be changed in order to contain the maximum fraction of BDs that is still marginally consistent (at the $10 \%$ level, i.e. $P_{\mathrm{KS}}=0.10$ ) with the observed JLF? There are two ways in which the Kroupa IMF can be changed: the first possibility is to change the power-law slope in the substellar regime, until the KS test between the model JLF to the observed cluster JLF yields $P_{\mathrm{KS}}=0.1$. We find that the slope must be changed from -0.3 to +2.5 . The corresponding IMF model (called "Fit I" in Table 2) has a BD fraction of 10\% (see Table 1).

The second possibility is to keep the power-law slope fixed at -0.3 and to increase the mass at which the power-law slope changes from -1.3 to -0.3 . For this alternative we find that the mass has to be increased from $0.08 M_{\odot}$ to $0.22 M_{\odot}$ (model "Fit II" in Table 2); the BD fraction of this model is $16 \%$.

Both models are compared to the observed cluster JLF in Fig. 5. This analysis gives an upper limit to the BD fraction of $<16 \%$ in IC 348 .

\subsection{Comparison to other IMF determinations for IC 348}

Lada et al. (1998) presented a study of the mass function of IC 348 based on the $K$-band luminosity function (KLF) of the

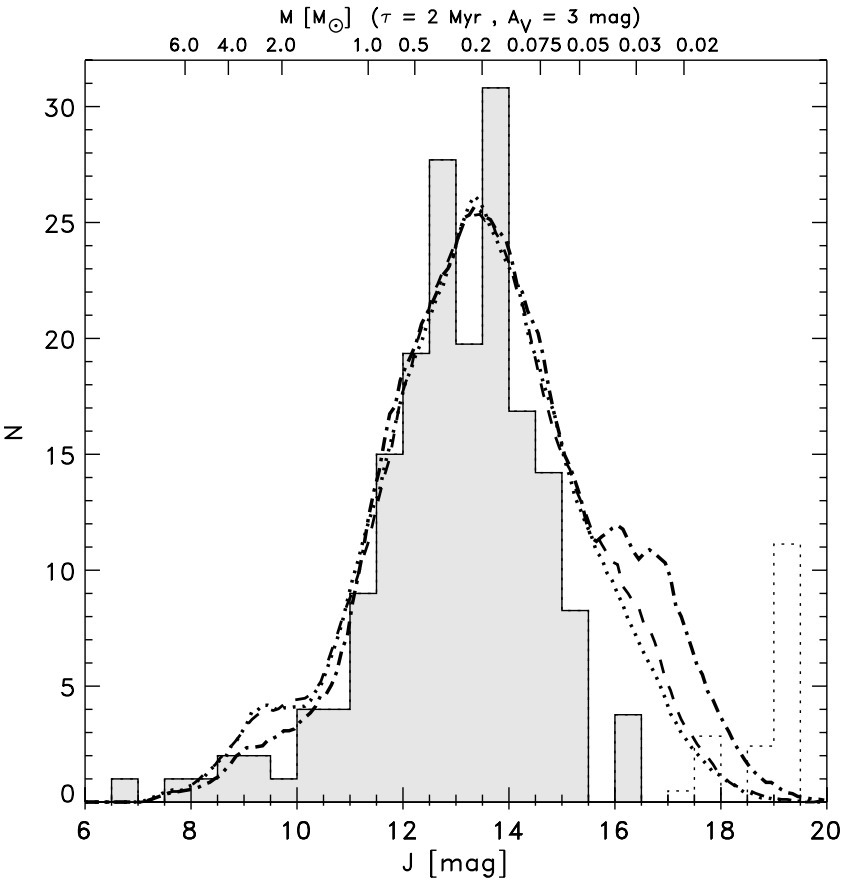

Fig. 7. The IC 348 JLF (histogram) compared to three models for the IMF in the Orion Trapezium Cluster. The dotted line shows the model by Hillenbrand \& Carpenter (2000), the dashed line shows the model by Luhman et al. (2000), and the dashed-dotted line the model by Muench et al. (2002).

cluster. The completeness limit of their KLF is near $K \sim 14$. Since the typical color of late M-type stars is $J-K \sim 1$, this corresponds to $J \sim 15$, i.e. their data are about 4 mag less deep than our $J$-band data. In their modeling, they consider two specific models which they find to be consistent with their data. The formulas for both models, called here L1 and L2, are given in Table 2, the model JLFs are compared to the observed cluster JLF in Fig. 6. Model L1 has a BD fraction of $19 \%$ and does not provide an acceptable description of our data. Model L2 has a BD fraction of $16 \%$ and provides a marginally acceptable fit to the JLF of IC 348. Both models predict more faint objects than observed. The deviations start at $J \sim 15$, i.e. just at the completeness limit of the $K$-band data used by Lada et al. (1998). We suspect that this explains why these models are consistent with their $K$-band data but not with our $J$-band data.

Luhman et al. (2000) determined an IMF for IC 348 based on a spectroscopic sample of cluster members. The simulated JLF based on this model (see Table 1 for details) is also shown in Fig. 6 and yields good agreement with the observed JLF. The model has a BD fraction of $10 \%$.

\subsection{IC 348 compared to the Orion Trapezium Cluster}

There are several reasons why it is interesting to compare the IMF of IC 348 to that of the Orion Trapezium Cluster. First, this famous star forming region is very well investigated, the stellar population is well known to a high degree of completeness, and several recent studies have tried to derive the IMF of this region. Second, the physical conditions in IC 348 are very different from those in the Trapezium Cluster and this might 
Table 2. Summary of model parameters and test statistics for all IMF models considered in this paper. For details see text. $P_{\mathrm{KS}}$ values above 0.1 , indicating statistically acceptable models, are marked in bold face.

\begin{tabular}{|c|c|c|c|c|c|c|c|}
\hline model & $\begin{array}{l}\text { mass } \mathrm{r} \\
\text { power- }\end{array}$ & $\begin{array}{l}\text { ange }\left[M_{\odot}\right] \\
\text { aw exponent }\end{array}$ & & & & $P_{\mathrm{KS}}$ & $\frac{N(0.02-0.075)}{N(>0.02)}$ \\
\hline $\begin{array}{l}\text { Kroupa (2002) } \\
\text { galactic field }\end{array}$ & $\begin{array}{c}{[0.02-0.08]} \\
\alpha=-0.3\end{array}$ & $\begin{array}{c}{[0.08-0.5]} \\
\alpha=-1.3\end{array}$ & $\begin{array}{c}{[0.5-100.0]} \\
\alpha=-2.3\end{array}$ & & & $4.12 \times 10^{-10}$ & 0.30 \\
\hline $\begin{array}{l}\text { Kroupa (2002) } \\
\text { stars only }\end{array}$ & $\begin{array}{c}{[0.08-0.5]} \\
\alpha=-1.3\end{array}$ & $\begin{array}{c}{[0.5-100.0]} \\
\alpha=-2.3\end{array}$ & & & & 0.97 & 0 \\
\hline $\begin{array}{l}\text { Chabrier (2002) } \\
\text { galactic field }\end{array}$ & $\operatorname{peak}(M)=0.1$ & $M_{\odot}, \sigma(\log M)=$ & $=0.627$ & & & $1.80 \times 10^{-12}$ & 0.34 \\
\hline $\log$-norm fit & $\operatorname{peak}(M)=0.2$ & $M_{\odot}, \sigma(\log M)$ & $=0.4$ & & & 0.78 & 0.09 \\
\hline $\begin{array}{l}\operatorname{maximum} \text { BD } \\
\text { Fit I }\end{array}$ & $\begin{array}{c}{[0.02-0.08]} \\
\alpha=+2.5\end{array}$ & $\begin{array}{c}{[0.08-0.5]} \\
\alpha=-1.3\end{array}$ & $\begin{array}{c}{[0.5-100.0]} \\
\alpha=-2.3\end{array}$ & & & 0.10 & 0.10 \\
\hline $\begin{array}{l}\text { maximum BD } \\
\text { Fit II }\end{array}$ & $\begin{array}{c}{[0.02-0.22]} \\
\alpha=-0.3\end{array}$ & $\begin{array}{l}{[0.2-0.5]} \\
\alpha=-1.3\end{array}$ & $\begin{array}{c}{[0.5-100.0]} \\
\alpha=-2.3\end{array}$ & & & 0.10 & 0.16 \\
\hline $\begin{array}{l}\text { Lada et al. (1998) } \\
\text { IC } 348 \text { (L1) }\end{array}$ & $\begin{array}{c}{[0.02-0.3]} \\
\alpha=-0.6\end{array}$ & $\begin{array}{l}{[0.3-1.0]} \\
\alpha=-1.4\end{array}$ & $\begin{array}{l}{[1.0-10]} \\
\alpha=-2.5\end{array}$ & & & $1.26 \times 10^{-2}$ & 0.19 \\
\hline $\begin{array}{l}\text { Lada et al. (1998) } \\
\text { IC } 348 \text { (L2) }\end{array}$ & $\begin{array}{c}{[0.02-0.2]} \\
\alpha=-0.3\end{array}$ & $\begin{array}{l}{[0.2-1.0]} \\
\alpha=-1.4\end{array}$ & $\begin{array}{l}{[1.0-10]} \\
\alpha=-2.5\end{array}$ & & & 0.11 & 0.16 \\
\hline $\begin{array}{l}\text { Luhman et al. (2000) } \\
\text { IC } 348\end{array}$ & $\begin{array}{c}{[0.02-0.1]} \\
\alpha=+0.4\end{array}$ & $\begin{array}{l}{[0.1-0.3]} \\
\alpha=-0.4\end{array}$ & $\begin{array}{l}{[0.3-2.5]} \\
\alpha=-2.0\end{array}$ & $\begin{array}{l}{[2.5-10]} \\
\alpha=-2.7\end{array}$ & & 0.86 & 0.10 \\
\hline $\begin{array}{l}\text { Hillenbrand \& } \\
\text { Carpenter (2000) } \\
\text { Trapezium Cluster }\end{array}$ & $\begin{array}{c}{[0.02-0.15]} \\
\alpha=-0.45\end{array}$ & $\begin{array}{l}{[0.15-0.4]} \\
\alpha=-1.22\end{array}$ & $\begin{array}{c}{[0.4-1.0]} \\
\alpha=-1.62\end{array}$ & $\begin{array}{c}{[1-3]} \\
\alpha=-1.88\end{array}$ & $\begin{array}{c}{[3-10]} \\
\alpha=-2.7\end{array}$ & $3.20 \times 10^{-3}$ & 0.21 \\
\hline $\begin{array}{l}\text { Luhman et al. (2000) } \\
\text { Trapezium Cluster }\end{array}$ & $\begin{array}{c}{[0.02-0.06]} \\
\alpha=-0.83\end{array}$ & $\begin{array}{c}{[0.06-0.08]} \\
\alpha=+0.1\end{array}$ & $\begin{array}{c}{[0.08-0.3]} \\
\alpha=-0.8\end{array}$ & $\begin{array}{l}{[0.3-2.5]} \\
\alpha=-1.7\end{array}$ & $\begin{array}{l}{[2.5-10]} \\
\alpha=-2.7\end{array}$ & $7.45 \times 10^{-4}$ & 0.22 \\
\hline $\begin{array}{l}\text { Muench et al. (2002) } \\
\text { Trapezium Cluster }\end{array}$ & $\begin{array}{c}{[0.02-0.025]} \\
\alpha=-6.00\end{array}$ & $\begin{array}{c}{[0.025-0.12]} \\
\alpha=-0.27\end{array}$ & $\begin{array}{l}{[0.12-0.6]} \\
\alpha=-1.15\end{array}$ & $\begin{array}{c}{[0.6-} \\
\alpha=-\end{array}$ & & $4.61 \times 10^{-7}$ & 0.27 \\
\hline
\end{tabular}

offer interesting clues concerning the reasons of IMF variations. We will consider several recent IMF determinations for the Trapezium Cluster and compare them to our IC 348 data.

Hillenbrand \& Carpenter (2000) presented the results of a deep near-infrared imaging survey of a $5^{\prime} \times 5^{\prime}$ central region of the Orion Trapezium Cluster. Their survey is sensitive to objects with masses down to $0.02 M_{\odot}$ and they use their data to constrain the shape of the stellar mass function. They found that the mass function rises to a peak around $0.15 M_{\odot}$ and then declines (in terms of $\mathrm{d} N / \mathrm{d}(\log M)$ ) across the hydrogen-burning limit. The parameterization of their mass function is given in Table 2, the resulting JLF is shown in Fig. 7. This model provides no valid description of the observed JLF for IC 348, because it predicts too many objects for $J \geq 14$ and generally produces a broader shaped JLF than observed. The KS test confirms that this model, which yields a BD fraction of $21 \%$, is not consistent with the IMF of IC 348.

Luhman et al. (2000) presented a study of the IMF in the Trapezium cluster based on HST/NICMOS data and groundbased $K$-band spectra. They constructed an HR diagram to determine stellar masses and ages. The IMF they derive is listed in Table 2 and the corresponding model JLF is shown in Fig. 7. The model JLF is very similar to the Hillenbrand \& Carpenter (2000) model; it has a BD fraction of $22 \%$ and also is inconsistent with the IMF of IC 348.

Muench et al. (2002) estimated the Trapezium cluster IMF from the observed $K$-band luminosity function. Their derived Trapezium cluster IMF (in the parameterization of their Eq. (1)) 


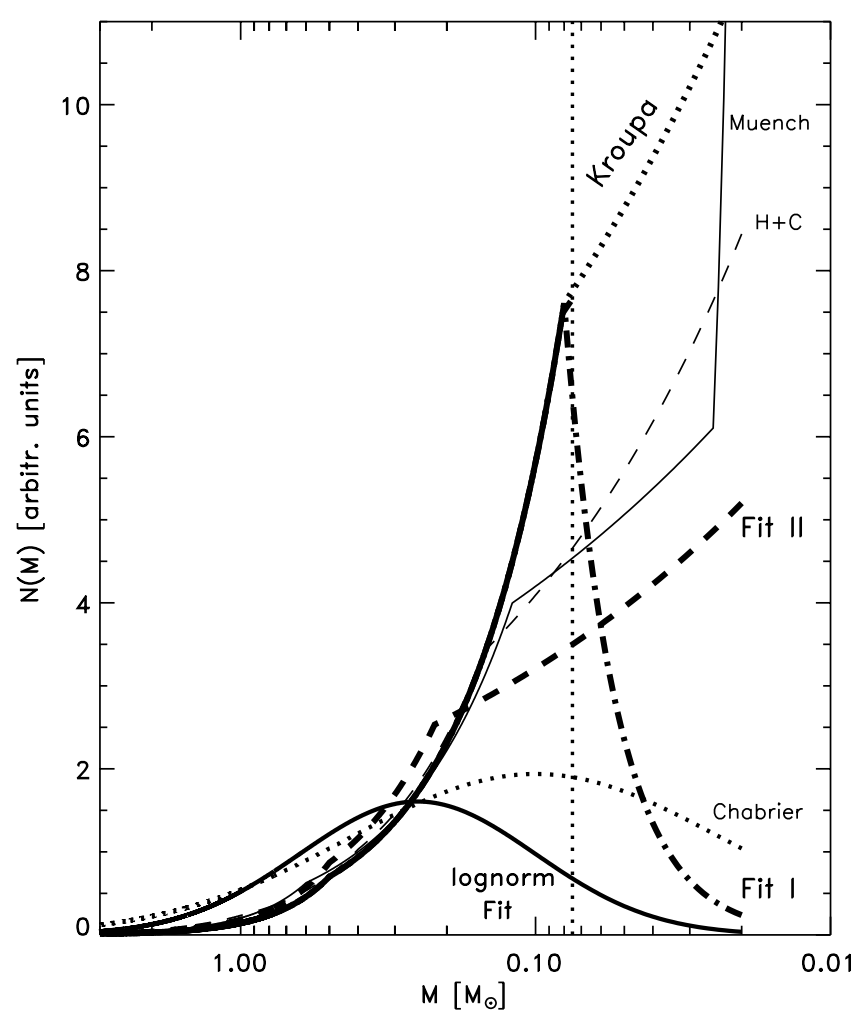

Fig. 8. Illustration of some of the IMFs used in this paper. All distributions are normalized to give the same number of objects in the 0.08-1.0 $M_{\odot}$ range. The thick solid line shows the Kroupa (2002) field star IMF, the sub-stellar part of which is shown by the thick dotted line. The thick dashed-dotted line shows the IMF "maximum BD fit I", the thick dashed line shows the IMF "maximum BD fit II". The solid parabolic line show the lognormal fit IMF, the dotted parabolic line represent the Chabrier (2002) field IMF. The thin solid line shows the Trapezium cluster IMF as derived by Muench et al. (2002), while the thin dashed line shows the Trapezium cluster IMF as derived by Hillenbrand \& Carpenter (2000).

is listed in Table 2. Their model has a BD fraction of $27 \%$ and the model JLF is obviously inconsistent with the JLF of IC 348.

\subsection{Conclusions drawn from the JLF modeling}

Our modeling shows that IC 348 harbors some BDs, but the size of the sub-stellar population is relatively small. The substellar part of the IMF in IC 348 is clearly different from that of the galactic field population and also the Orion Trapezium cluster. With a BD fraction of $\sim 10 \%$ (at most $16 \%$ ) the substellar population (in the $0.02-0.075 M_{\odot}$ mass range) in IC 348 is about two times smaller than in the galactic field and the Trapezium cluster which have BD fractions of about $20-35 \%$.

\subsection{Comparison to the BD deficit in Taurus}

It is very interesting to note that recent results suggest a similar deficit of BDs for the Taurus star forming region. Luhman (2000) investigated the IMF of the Taurus star forming region and found strong evidence for a significant deficit of BDs in that region. This result was recently confirmed and strengthened in the study by Briceno et al. (2002), who concluded that Taurus has $2 \times$ fewer brown dwarfs at $0.02-0.08 M_{\odot}$ than the Orion Trapezium cluster.

Our results on IC 348 therefore provide another piece of evidence for the non-universality of the sub-stellar IMF.

\section{Possible explanations for the BD deficit}

How can we understand the observed differences in the substellar IMF of IC 348 (and similarly Taurus) as compared to the galactic field population and the Orion Trapezium Cluster? There are a number of important differences in the physical environment of IC 348 as compared to the Trapezium Cluster. One crucial factor may be the density in the star forming environment. The stellar number density of $n_{\star} \sim 10^{3} \mathrm{pc}^{-3}$ in IC 348 is much lower than the density of $n_{\star} \sim 5 \times 10^{4} \mathrm{pc}^{-3}$ in the central Trapezium cluster. Briceno et al. (2002) argued that in low-density star forming environments the minimum Jeans mass may be larger, possibly explaining the BD deficit in Taurus and perhaps also in IC 348. Another important factor may lie in the different levels of supersonic turbulence in the molecular cloud, which influences the star formation process (see Mac Low \& Klessen 2003 and also the discussion by Luhman 2000).

A very critical aspect we want to dicuss in some more detail is the presence or absence of massive $\left(M \geq 10 M_{\odot}\right)$ stars and their impact on the forming young stellar objects in their vicinity. In IC 348, the most massive star is only a B5 V star ${ }^{1}$ with $\sim 6 M_{\odot}$, which neither has a powerful wind nor emits strong ionizing radiation. In the Trapezium cluster, on the other hand, the strong winds and the ionizing radiation of the massive stars obviously affect nearby collapsing cloud cores and forming stars, for example by dispersing the circumstellar material of young stellar objects, as can be nicely seen in the ionized proplyds (Bally et al. 1998; see also Richling \& Yorke 1998). The mass loss rates of these proplyds due to photoevaporation have been estimated to be $\approx 10^{-6} M_{\odot} /$ yr (Henney \& O'Dell 1999), and recent numerical simulations suggest that even higher evaporation rates of up to $\approx 10^{-5} M_{\odot} / \mathrm{yr}$ can be reached next to massive stars (cf. Brandner et al. 2000; Hollenbach et al. 2000). The final mass of the forming young stellar objects is therefore strongly influenced by the competition between accretion and photoevaporation. This scenario was recently explored in some detail by Whitworth \& Zinnecker (2003; see also Kroupa 2001, 2002). They found that the erosion of initially more massive pre-stellar cores by the ionizing radiation from ambient massive stars can significantly diminish the accretion reservoir. In the central regions of large clusters the outer layers of the cores are eroded more rapidly than they can accrete onto a central protostar, and this can lead to the formation of BDs instead of low-mass stars. In a cluster like IC 348, i.e. in the absence

\footnotetext{
1 We note that H98 detected a faint blue star (his number 209) in IC 348, for which the position in the color-magnitude diagram suggest that it might be a white dwarf. It is not known whether this star is a member to IC 348 or a background object; if it really is a member of the cluster, there may have been one massive star in IC 348 at one time.
} 
of massive stars, the pre-stellar cores are unaffected by photoerosion and the protostellar objects should therefore reach systematically higher final masses; thus, a larger fraction of the forming very-low-mass objects can gain enough mass to cross the hydrogen-burning limit.

Another interesting aspect in this context is the result that the stellar part of the IMF in IC 348 is very similar to the field IMF, whereas the number of BDs is much smaller than in the field. This may be understood in the framework of the theory by Elmegreen (2000) that the IMF is the combination of two independent mass functions that combine in different ways above and below a characteristic mass. In the intermediate- to highmass range the IMF depends primarily on the cloud structure, which seems to be rather universal and leads to very similar mass functions independent of the details of the star formation process. The turnover and the flat or declining part of the IMF is determined by the details of the transition from clumps to stars and should be sensitive to the physical conditions during the star formation process. Since the presence (or absence) of massive stars seriously affects the physical conditions during the star formation process, this would yield an interesting explanation for variations in the very-low-mass IMF.

However, it is unclear whether the impact of massive stars actually can fully explain the observed differences in the substellar populations in various environments. One problem is that photoevaporation significantly affects only the low-mass objects in the immediate vicinity of the most massive stars, whereas objects in the outer part of the cluster are not so strongly influenced (Whitworth \& Zinnecker 2003). Secondly, this scenario requires that the massive stars have already entered their main-sequence phase while the low-mass objects are still in a very early evolutionary phase. This may be a particularly serious problem if the hypothesis of a very recent birth for $\theta^{1} \mathrm{C}$ Ori (probably within the last few 10000 years; see e.g. Scally \& Clarke 2001) is correct.

Therefore, we finally also consider a totally different scenario. It has been suggested that BDs can form via the fragmentation of dense molecular gas in unstable multiple systems and are ejected from the dense gas before they have been able to accrete to stellar masses (Reipurth \& Clarke 2001; Bate et al. 2002). In the simulations of Bate et al. (2002), this formation mechanism produced roughly the same number of BDs than stars and therefore reproduces the observed size of the BD population in the galactic field. According to this scenario, typical ejection velocities are about $3-5 \mathrm{~km} \mathrm{~s}^{-1}$. In a $2 \mathrm{Myr}$ period (the age of IC 348) the ejected BDs would therefore move up to about $10 \mathrm{pc}$ and would be displaced up to $\sim 2^{\circ}$ from their formation site near the cluster center. One would therefore expect that a large fraction of the BD population has already left the cluster area and is widely dispersed.

In the Trapezium cluster, on the other hand, one would expect to see a larger BD population for two reasons: First, the cluster is younger ( $\sim 1 \mathrm{Myr})$ than IC 348 , therefore some of the ejected BDs have not yet moved far away. Second, due to the higher total mass of the Orion Trapezium cluster $\left(\sim 1100 M_{\odot}\right.$, cf. Lada \& Lada 2003 , versus $\sim 110 M_{\odot}$ for IC 348) and its much higher central density $\left(n_{*} \sim 47000 \mathrm{pc}^{-3}\right.$ for the central $0.1 \mathrm{pc}$ region in the Trapezium cluster, cf. McCaughrean \& Stauffer 1994, versus $n_{*} \sim 4200 \mathrm{pc}^{-3}$ for the central $0.1 \mathrm{pc}$ region of IC 348; cf. Lada \& Lada 1995) it is harder for ejected bodies to leave the much deeper gravitational potential. The mean escape velocity for IC 348 is $\sim 0.8 \mathrm{~km} \mathrm{~s}^{-1}$ (Herbig 1998), while the corresponding value for the Orion Trapezium cluster is $\sim 2 \mathrm{~km} \mathrm{~s}^{-1}$. The statistical analysis of dynamical interactions during early cluster evolution of Sterzik $\&$ Durisen (2003) suggests that the median velocities of BDs are $\lesssim 2 \mathrm{~km} \mathrm{~s}^{-1}$; this would imply that in IC 348 a large fraction of the BDs could be ejected from the cluster, while in the Orion Trapezium cluster most of the BDs would be gravitationally bound. We therefore conclude that the ejection scenario might offer a good explanation for the observed differences in the sizes of substellar populations. A direct proof for this theory, however, can only be obtained via a detailed investigation of the dynamical status of the stellar and sub-stellar cluster populations. This would require to measure the proper motions of objects with an accuracy of the order of $1 \mathrm{~km} \mathrm{~s}^{-1}$. While such a project is hardly feasible with current technology, we note that it will soon be possible to obtain wide-field diffraction limited astrometric imaging with the LBT interferometer (see Zinnecker \& McCaughrean 2001); in this way the validity of the ejection model could be tested.

\section{Summary}

Our modeling of the observed JLF in IC 348 shows that the stellar part of the IMF agrees well with the field star IMF. We find evidence that the BD population in IC 348 is about 2-3 times smaller than in the general field and is also about 2 times smaller than in the Orion Trapezium cluster. The deficit of BDs in IC 348 appears to be in line with a similar BD deficit recently found for the Taurus star forming region. These results provide evidence for the non-universality of the sub-stellar IMF and challenge current theories of very low-mass star formation and early dynamical evolution.

\section{Final note}

After completion of this work, we became aware of a recent paper by Muench et al. (2003), in which a similar near-infrared investigation of the luminosity- and mass function of IC 348 was presented. They find that the IMF of IC 348 decreases in a much steeper manner than the Trapezium IMF (as derived by Muench et al. 2002) and that the objects in the 0.025-0.08 $M_{\odot}$ mass range constitute only $\sim 14 \%$ of the members in IC 348 , considerably less than in the Trapezium. These results agree quite well with our findings. However, in distinction to our study, they find indications for a secondary peak in the IMF of IC 348, similar to that reported by Muench et al. (2002) for the Trapezium IMF, which may increase the substellar fraction for IC 348.

Very recently, Luhman et al. (2003, [astro-ph/0304409]) presented a new census of the stellar and substellar members of IC 348. From spectroscopy of candidate cluster members they identified numerous new members, including several BDs. Their final sample of 288 spectroscopically identified cluster 
members in IC 348 contains 23 BDs. The fraction of BDs in their sample, $\sim 8 \%$, is in good agreement with our results.

Acknowledgements. We would like to thank the Calar-Alto staff for carrying out the observations in service mode (Observer: Ana Guijarro). We thank George H. Herbig for helpful comments and interesting discussion about IC 348. Th. P. would like to thank George $\mathrm{H}$. Herbig and the other members of the Institute for Astronomy at the University of Hawaii for their kind hospitality during his visits at the Institute and for useful discussions on IC 348. This publication makes use of data products from the Two Micron All Sky Survey, which is a joint project of the University of Massachusetts and the Infrared Processing and Analysis Center/California Institute of Technology, funded by the National Aeronautics and Space Administration and the National Science Foundation.

\section{References}

Babu, G. J., \& Feigelson, E. D. 1996, Astrostatistics (London: Chapman \& Hall)

Bally, J., Sutherland, R. S., Devine, D., \& Johnstone, D. 1998, AJ, 116, 293

Bate, M. R., Bonnell, I. A., \& Bromm, V. 2002, MNRAS, 332, L65

Baraffe, I., Chabrier, G., Allard, F., \& Hauschildt, P. H. 1998, A\&A, 337,403

Baraffe, I., Chabrier, G., Barman, T. S., Allard, F., \& Hauschildt, P. H. 2003, A\&A, 402, 701

Bizenberger, P., McCaughrean, M. J., Birk, C., Thompson, D., \& Storz, C. 1998, Proc. SPIE, 3354, 825

Brandnder, W., Grebel, E. K., Chu, Y.-H., et al. 2000, AJ, 119, 292

Briceno, C., Luhmann, K. L., Hartmann, L., Stauffer, J. R., \& Kirkpatrick, J. D. 2002, ApJ, 580, 317

Burrows, A., Marley, M., Hubbard, W. B., et al. 1997, ApJ, 491, 856

Cernicharo, J., Bachiller, R., \& Duvert, G. 1985, A\&A, 149, 273

Cernis, K. 1993, Baltic Astron., 2, 214

Chabrier, G. 2002, ApJ, 567, 304

Chabrier, G., \& Baraffe, I. 2000, ARA\&A, 38, 337

D’Antona, F., \& Mazzitelli, I. 1997, in Cool stars in Clusters and Associations, ed. R. Pallavicini \& G. Micela, Mem. S. A. It., 68, 4

de Zeeuw, P. T., Hoogerwerf, R., de Bruijne, J. H. J., Brown, A. G. A., \& Blaauw, A. 1999, AJ, 117, 354

Duchene, G., Bouvier, J., \& Simon, T. 1999, A\&A, 343, 831

Duquennoy, A., \& Mayor, M. 1991, A\&A, 248, 485

Eislöffel, J., Fröbrich, D., Stanke, T., \& McCaughrean, M. J. 2003, ApJ, submitted

Elmegreen, B. G. 2000, MNRAS, 311, L5

Fischer, D., \& Marcy, G. 1992, ApJ, 396, 178

Harris, D. L., Morgan, W. W., \& Roman, N. G. 1954, ApJ, 119, 622

Henney, W. J., \& O'Dell, C. R. 1999, AJ, 118, 2350

Herbig, G. H. 1954, PASP, 66, 19

Herbig, G. H. 1998, ApJ, 497, 736 [H98]

Hillenbrand, L. A., \& Carpenter, J. M. 2000, ApJ, 540, 236

Hollenbach, D. J., Yorke, H. W., Johnstone, D. 2000, in Protostars and Planets IV, ed. V. Mannings, A. P. Boss, \& S. S. Russell (Tucson: University of Arizona Press), 401

Kenyon, S. J., \& Hartmann, L. 1995, ApJS, 101, 117

Kroupa, P. 2001, MNRAS, 322, 231

Kroupa, P. 2002, Science, 295, 82

Lada, E. A., \& Lada, C. J. 1995, AJ, 109, 1682
Lada, E. A., \& Lada, C. J. 2003, ARA\&A, in press

Lada, E. A., Lada, C. J., \& Muench, A. 1998, in The Stellar Initial Mass Function, ed. G. Gilmore, D. Howell, ASP Conf. Ser., 142, 107

Leggett, S. K., Allard, F., Berriman, G., Dahn, C. C., Hauschildt, P. H. 1996, ApJS, 104, 117

Liu, M. C. 2002, in The twelfth Cambridge Workshop on Cool Stars, Stellar Systems and the Sun, ASP Conf. Ser., in press

Luhman, K. L., Rieke, G. H., Lada, C. J., \& Lada, E. A. 1998, ApJ, 508, 347 (L98)

Luhman, K. L. 1999, ApJ, 525, 466

Luhman, K. L. 2000, ApJ, 544, 1044

Luhman, K. L., Rieke, G. H., Young, E. T., et al. 2000, ApJ, 540, 1016 Mac Low, M.-M., \& Klessen, R. S. 2003, Rev. Mod. Phys, in press

Martin, E., et al. 2003, in IAU Symp. 211: Brown Dwarfs, ASP Conf. Ser., in press

McCaughrean, M. J., \& Stauffer, J. R. 1994, AJ, 108,1382

McCaughrean, M. J., Rayner, J. T., \& Zinnecker, H. 1994, ApJ, 436, L189

McCaughrean, M. J., Zinnecker, H., Rayner, J. T., \& Stauffer, J. 1995, in The bottom of the main sequence - and beyond, ed. C. G. Tinney, ESO Astrophys. Symp. (Springer Verlag), 209

Miller G. E., \& Scalo J. M. 1979, ApJS, 41, 513

Muench, A. A., Lada, E. A., Lada, C. J., \& Alves, J. 2002, ApJ, 573, 366

Muench, A. A., Lada, E. A., Lada, C. J., et al. 2003, AJ, 125,2029

Najita, J. R., Tiede, G. P., \& Carr, J. S. 2000, ApJ, 541, 977 [N00]

Oppenheimer, B. R., Kulkarni, S. R., Matthews, K., Nakajima, T. 1995, Science, 270, 1478

Oppenheimer, B. R., Kulkarni, S. R., \& Stauffer, J. R. 2000, in Protostars and Planets IV, ed. V. Mannings, A. P. Boss, S. S. Russell (Tucson: University of Arizona Press), 1313

Palla, F., \& Stahler S. W. 1999, ApJ, 525,772

Preibisch, Th., \& Zinnecker, H. 1999, AJ, 117, 2381

Preibisch, Th., \& Zinnecker, H. 2001, AJ, 122, 866

Preibisch, Th., \& Zinnecker, H. 2002, AJ, 123, 1613

Preibisch, Th., Brown, A., Bridges, T., Guenther, E., \& Zinnecker, H. 2002, AJ, 124, 404

Preibisch, Th., Zinnecker, H., \& Herbig, G. H. 1996, A\&A, 310, 456

Rebolo, R., Zapatero Osori, M. R., \& Martin, E. L. 1995, Nature, 227, 129

Reid, I. N., Kirkpatrick, J. D., Liebert, J., et al. 1999, ApJ, 521, 613

Reipurth, B., \& Clarke, C. 2001, AJ, 122, 432

Richling, S., \& Yorke, H. W. 1998, A\&A, 340, 408

Rieke, G. H., \& Lebofsky, M. J. 1985, ApJ, 288, 618

Ripepi, V., Palla, F., Marconi, M., et al. 2002, A\&A, 391, 587

Scally, A., \& Clarke, C. 2001, MNRAS, 325, 449

Scalo, J. 1998, in The Stellar Initial Mass Function, ed. G. Gilmore, D. Howell, ASP Conf. Ser., 142, 201

Schlegel, D. J., Finkenbeiner, D. P., \& Davis, M. 1998, ApJ, 500, 525

Sterzik, M. F., \& Durisen, R. H. 2003, A\&A, 400, 1031

White, R. J., Ghez, A. M., Reid, I. N., \& Schultz, G. 1999, ApJ, 520, 811

Whitworth, A., \& Zinnecker, H. 2003, A\&A, submitted

Yorke, H. W. 1986, ARA\&A, 24, 49

Zinnecker, H., \& McCaughrean, M. J. 2001, in Science with the Large Binocular Telescope, ed. T. Herbst, p. 137

Zinnecker, H., McCaughrean, M. J., \& Wilking, B. A. 1993, in Protostars and Planets III, ed. E. H. Levy, \& J. I. Lumine (University of Arizona Press), 429 


\section{Online Material}


T. Preibisch et al.: IMF and brown dwarf population of IC 348, Online Material $p 2$

Table 1. $J$-band magnitudes of the sources detected in our IC 348 image. The source names contain the J2000 coorinates.

\begin{tabular}{|c|c|}
\hline IC348-J & $\begin{array}{c}J \\
{[\mathrm{mag}]}\end{array}$ \\
\hline $034350.6+321059$ & 18.69 \\
\hline $034350.6+321231$ & 19.46 \\
\hline $034350.7+321306$ & 14.20 \\
\hline $034350.7+321125$ & 19.98 \\
\hline $034350.8+321433$ & 19.65 \\
\hline $034350.9+321133$ & 20.45 \\
\hline $034350.9+321429$ & 20.65 \\
\hline $034351.0+321513$ & 16.30 \\
\hline $034351.0+321127$ & 19.17 \\
\hline $034351.1+321139$ & 17.84 \\
\hline $034351.1+320701$ & 16.94 \\
\hline $034351.1+321359$ & 20.01 \\
\hline $034351.1+321348$ & 18.74 \\
\hline $034351.2+321525$ & 20.02 \\
\hline $034351.2+320928$ & 18.73 \\
\hline $034351.2+321045$ & 17.76 \\
\hline $034351.2+321430$ & 20.35 \\
\hline $034351.3+320656$ & 17.13 \\
\hline $034351.3+321309$ & 10.25 \\
\hline $034351.3+320720$ & 19.28 \\
\hline $034351.3+321154$ & 17.32 \\
\hline $034351.3+320812$ & 19.69 \\
\hline $034351.4+320504$ & 17.80 \\
\hline $034351.4+321141$ & 19.66 \\
\hline $034351.4+321508$ & 20.63 \\
\hline $034351.4+320708$ & 19.16 \\
\hline $034351.4+321518$ & 17.81 \\
\hline $034351.5+321630$ & 20.41 \\
\hline $034351.5+321028$ & 20.02 \\
\hline $034351.6+320740$ & 19.87 \\
\hline $034351.6+320844$ & 18.74 \\
\hline $034351.6+321124$ & 16.72 \\
\hline $034351.6+321240$ & 20.01 \\
\hline $034351.6+321313$ & 19.33 \\
\hline $034351.6+320552$ & 18.14 \\
\hline $034351.6+321556$ & 12.99 \\
\hline $034351.7+321347$ & 18.70 \\
\hline $034351.7+320526$ & 19.91 \\
\hline $034351.8+320632$ & 19.09 \\
\hline $034351.9+320220$ & 20.20 \\
\hline $034351.9+320211$ & 20.04 \\
\hline $034351.9+321206$ & 20.71 \\
\hline $034351.9+321627$ & 19.99 \\
\hline $034352.0+321524$ & 19.72 \\
\hline $034352.0+321201$ & 16.96 \\
\hline $034352.0+320339$ & 13.79 \\
\hline $034352.0+320948$ & 18.04 \\
\hline $034352.0+321326$ & 19.45 \\
\hline $034352.0+320949$ & 19.34 \\
\hline $034352.1+321413$ & 18.94 \\
\hline $034352.2+321350$ & 20.14 \\
\hline $034352.3+320732$ & 18.60 \\
\hline $034352.3+321534$ & 19.31 \\
\hline $034352.3+321527$ & 20.67 \\
\hline $034352.3+321214$ & 20.17 \\
\hline $034352.4+321432$ & 21.19 \\
\hline $034352.4+321029$ & 20.11 \\
\hline
\end{tabular}

\begin{tabular}{|c|c|}
\hline IC348-J & $\begin{array}{c}J \\
{[\mathrm{mag}]}\end{array}$ \\
\hline $034352.4+321049$ & 19.93 \\
\hline $034352.5+321121$ & 20.87 \\
\hline $034352.5+320705$ & 19.57 \\
\hline $034352.6+320118$ & 19.62 \\
\hline $034352.7+320736$ & 18.83 \\
\hline $034352.7+320752$ & 20.13 \\
\hline $034352.7+321320$ & 20.15 \\
\hline $034352.7+321326$ & 19.07 \\
\hline $034352.7+320652$ & 18.40 \\
\hline $034352.7+321204$ & 18.15 \\
\hline $034352.8+321441$ & 18.09 \\
\hline $034352.8+320814$ & 18.42 \\
\hline $034352.8+321350$ & 19.67 \\
\hline $034352.8+321523$ & 19.47 \\
\hline $034352.8+320617$ & 19.97 \\
\hline $034352.8+320901$ & 18.89 \\
\hline $034353.1+321306$ & 17.40 \\
\hline $034353.1+320954$ & 19.55 \\
\hline $034353.2+320844$ & 14.57 \\
\hline $034353.2+321555$ & 18.14 \\
\hline $034353.3+321202$ & 15.58 \\
\hline $034353.3+320924$ & 19.32 \\
\hline $034353.3+321300$ & 14.07 \\
\hline $034353.4+320925$ & 17.91 \\
\hline $034353.5+320726$ & 16.20 \\
\hline $034353.5+321040$ & 19.77 \\
\hline $034353.5+321312$ & 17.47 \\
\hline $034353.5+321418$ & 15.78 \\
\hline $034353.6+321017$ & 16.48 \\
\hline $034353.6+321715$ & 20.09 \\
\hline $034353.6+321229$ & 20.02 \\
\hline $034353.7+320905$ & 17.99 \\
\hline $034353.7+321145$ & 19.49 \\
\hline $034353.7+320805$ & 20.10 \\
\hline $034353.8+321334$ & 19.14 \\
\hline $034353.8+321217$ & 17.57 \\
\hline $034353.8+320730$ & 13.68 \\
\hline $034353.9+321054$ & 16.70 \\
\hline $034353.9+320225$ & 19.75 \\
\hline $034353.9+321311$ & 19.82 \\
\hline $034353.9+321255$ & 20.11 \\
\hline $034354.0+321339$ & 18.29 \\
\hline $034354.0+320842$ & 18.60 \\
\hline $034354.1+320933$ & 18.88 \\
\hline $034354.2+320212$ & 18.04 \\
\hline $034354.2+321705$ & 20.18 \\
\hline $034354.2+320611$ & 19.59 \\
\hline $034354.2+321147$ & 16.71 \\
\hline $034354.3+321333$ & 20.35 \\
\hline $034354.3+320148$ & 20.22 \\
\hline $034354.4+320242$ & 20.02 \\
\hline $034354.5+321327$ & 19.28 \\
\hline $034354.5+320210$ & 19.93 \\
\hline $034354.5+321014$ & 13.25 \\
\hline $034354.6+321249$ & 17.30 \\
\hline $034354.6+320754$ & 19.56 \\
\hline $034354.6+320853$ & 19.73 \\
\hline
\end{tabular}

\begin{tabular}{|c|c|}
\hline IC348-J & $\begin{array}{c}J \\
{[\mathrm{mag}]}\end{array}$ \\
\hline $034354.6+320853$ & 19.73 \\
\hline $034354.6+320723$ & 17.47 \\
\hline $034354.7+320912$ & 19.64 \\
\hline $034354.7+321320$ & 20.31 \\
\hline $034354.7+320856$ & 18.26 \\
\hline $034354.7+321210$ & 17.02 \\
\hline $034354.8+321129$ & 17.50 \\
\hline $034354.8+321340$ & 17.27 \\
\hline $034354.9+320929$ & 20.16 \\
\hline $034354.9+321427$ & 19.53 \\
\hline $034355.0+321211$ & 19.18 \\
\hline $034355.1+321426$ & 18.51 \\
\hline $034355.1+320714$ & 14.10 \\
\hline $034355.1+321326$ & 15.89 \\
\hline $034355.1+321612$ & 19.72 \\
\hline $034355.2+321006$ & 16.37 \\
\hline $034355.3+320154$ & 19.90 \\
\hline $034355.3+320753$ & 13.57 \\
\hline $034355.3+321402$ & 19.60 \\
\hline $034355.3+321700$ & 17.88 \\
\hline $034355.4+321102$ & 20.25 \\
\hline $034355.4+321655$ & 19.74 \\
\hline $034355.4+321249$ & 19.70 \\
\hline $034355.5+320704$ & 17.98 \\
\hline $034355.5+321001$ & 19.22 \\
\hline $034355.5+320931$ & 11.28 \\
\hline $034355.6+320956$ & 20.03 \\
\hline $034355.6+321451$ & 18.54 \\
\hline $034355.6+321343$ & 20.20 \\
\hline $034355.6+321530$ & 16.32 \\
\hline $034355.6+321325$ & 16.81 \\
\hline $034355.7+321701$ & 18.75 \\
\hline $034355.7+320912$ & 19.83 \\
\hline $034355.7+321504$ & 20.14 \\
\hline $034355.9+321603$ & 19.68 \\
\hline $034355.9+320921$ & 19.18 \\
\hline $034356.0+320212$ & 12.34 \\
\hline $034356.0+320928$ & 19.83 \\
\hline $034356.0+320943$ & 20.09 \\
\hline $034356.1+320306$ & 19.66 \\
\hline $034356.1+320734$ & 19.91 \\
\hline $034356.1+321443$ & 19.79 \\
\hline $034356.2+321403$ & 17.85 \\
\hline $034356.2+321605$ & 18.07 \\
\hline $034356.2+321255$ & 19.11 \\
\hline $034356.2+320836$ & 12.58 \\
\hline $034356.4+320959$ & 15.38 \\
\hline $034356.4+321633$ & 19.89 \\
\hline $034356.5+321516$ & 19.77 \\
\hline $034356.5+321506$ & 18.20 \\
\hline $034356.6+321325$ & 19.87 \\
\hline $034356.6+321114$ & 19.55 \\
\hline $034356.6+321720$ & 19.99 \\
\hline $034356.7+321516$ & 16.95 \\
\hline $034356.7+321039$ & 19.25 \\
\hline $034356.7+320721$ & 19.46 \\
\hline $034356.7+321510$ & 19.17 \\
\hline
\end{tabular}


T. Preibisch et al.: IMF and brown dwarf population of IC 348, Online Material $p 3$

Table 1. continued.

\begin{tabular}{|c|c|}
\hline IC348-J & $\begin{array}{c}J \\
{[\mathrm{mag}]}\end{array}$ \\
\hline $034356.9+321316$ & 19.40 \\
\hline $034356.9+321415$ & 17.66 \\
\hline $034356.9+321522$ & 20.20 \\
\hline $034356.9+320534$ & 19.86 \\
\hline $034356.9+321428$ & 16.79 \\
\hline $034357.0+321400$ & 19.92 \\
\hline $034357.0+321434$ & 19.87 \\
\hline $034357.2+320133$ & 15.72 \\
\hline $034357.3+321258$ & 12.75 \\
\hline $034357.3+320805$ & 20.01 \\
\hline $034357.4+321632$ & 19.85 \\
\hline $034357.5+321454$ & 19.80 \\
\hline $034357.5+321711$ & 19.31 \\
\hline $034357.5+321551$ & 19.46 \\
\hline $034357.6+321508$ & 19.34 \\
\hline $034357.6+320137$ & 14.60 \\
\hline $034357.6+321449$ & 14.87 \\
\hline $034357.6+321320$ & 17.46 \\
\hline $034357.7+320914$ & 18.12 \\
\hline $034357.8+320750$ & 17.68 \\
\hline $034357.8+321158$ & 18.59 \\
\hline $034357.8+321619$ & 17.33 \\
\hline $034357.9+321544$ & 20.12 \\
\hline $034357.9+321234$ & 17.73 \\
\hline $034358.0+321048$ & 19.92 \\
\hline $034358.0+321144$ & 16.33 \\
\hline $034358.0+320633$ & 19.84 \\
\hline $034358.1+320946$ & 9.85 \\
\hline $034358.1+321556$ & 17.57 \\
\hline $034358.2+321357$ & 14.10 \\
\hline $034358.2+321432$ & 14.10 \\
\hline $034358.2+320946$ & 12.00 \\
\hline $034358.2+321015$ & 18.42 \\
\hline $034358.2+321239$ & 17.70 \\
\hline $034358.5+320835$ & 18.77 \\
\hline $034358.6+320959$ & 17.74 \\
\hline $034358.7+321509$ & 19.83 \\
\hline $034358.7+321517$ & 19.46 \\
\hline $034358.8+320830$ & 18.92 \\
\hline $034358.8+321225$ & 16.00 \\
\hline $034358.9+321053$ & 19.75 \\
\hline $034358.9+321127$ & 12.15 \\
\hline $034358.9+321023$ & 17.68 \\
\hline $034359.0+320958$ & 15.74 \\
\hline $034359.0+321715$ & 19.98 \\
\hline $034359.1+321421$ & 13.19 \\
\hline $034359.1+320829$ & 19.48 \\
\hline $034359.2+320556$ & 14.76 \\
\hline $034359.2+320933$ & 19.44 \\
\hline $034359.2+321430$ & 20.27 \\
\hline $034359.2+321450$ & 18.79 \\
\hline $034359.2+320250$ & 18.33 \\
\hline $034359.2+321402$ & 18.89 \\
\hline $034359.3+321705$ & 20.14 \\
\hline $034359.3+320625$ & 17.62 \\
\hline $034359.5+321505$ & 19.34 \\
\hline
\end{tabular}

\begin{tabular}{|c|c|}
\hline IC348-J & $\begin{array}{c}J \\
{[\mathrm{mag}]}\end{array}$ \\
\hline $034359.5+321555$ & 12.84 \\
\hline $034359.6+320604$ & 17.63 \\
\hline $034359.6+321143$ & 19.05 \\
\hline $034359.6+320154$ & 13.84 \\
\hline $034359.7+321549$ & 18.84 \\
\hline $034359.7+321455$ & 19.49 \\
\hline $034359.7+321403$ & 12.25 \\
\hline $034359.7+321559$ & 16.28 \\
\hline $034359.8+320921$ & 19.22 \\
\hline $034359.9+320441$ & 14.26 \\
\hline $034359.9+321047$ & 13.88 \\
\hline $034359.9+321115$ & 12.21 \\
\hline $034359.9+320908$ & 17.87 \\
\hline $034360.0+320740$ & 18.90 \\
\hline $034400.0+321328$ & 19.31 \\
\hline $034400.1+320654$ & 18.29 \\
\hline $034400.2+321548$ & 19.50 \\
\hline $034400.2+320655$ & 19.85 \\
\hline $034400.2+321458$ & 19.54 \\
\hline $034400.2+321354$ & 17.17 \\
\hline $034400.3+321132$ & 19.36 \\
\hline $034400.5+320432$ & 19.57 \\
\hline $034400.5+320742$ & 19.00 \\
\hline $034400.5+321058$ & 17.80 \\
\hline $034400.6+321521$ & 17.15 \\
\hline $034400.6+321344$ & 19.58 \\
\hline $034400.6+321207$ & 19.69 \\
\hline $034400.7+320419$ & 19.42 \\
\hline $034400.8+321107$ & 18.43 \\
\hline $034400.9+321240$ & 20.02 \\
\hline $034400.9+320818$ & 20.24 \\
\hline $034400.9+321442$ & 19.89 \\
\hline $034400.9+320538$ & 18.76 \\
\hline $034400.9+321056$ & 19.17 \\
\hline $034401.0+321350$ & 20.27 \\
\hline $034401.1+321532$ & 17.33 \\
\hline $034401.1+321648$ & 19.08 \\
\hline $034401.2+321058$ & 19.75 \\
\hline $034401.2+321230$ & 19.39 \\
\hline $034401.3+321341$ & 19.05 \\
\hline $034401.4+320549$ & 16.48 \\
\hline $034401.4+321022$ & 19.26 \\
\hline $034401.5+321553$ & 17.78 \\
\hline $034401.5+321450$ & 18.84 \\
\hline $034401.5+321541$ & 20.46 \\
\hline $034401.8+321632$ & 19.10 \\
\hline $034401.9+321253$ & 17.79 \\
\hline $034401.9+321121$ & 15.18 \\
\hline $034402.0+321426$ & 19.56 \\
\hline $034402.0+321536$ & 20.03 \\
\hline $034402.0+321001$ & 18.82 \\
\hline $034402.1+321621$ & 19.09 \\
\hline $034402.1+320615$ & 19.96 \\
\hline $034402.2+320731$ & 20.30 \\
\hline $034402.3+321430$ & 18.38 \\
\hline $034402.3+321128$ & 18.86 \\
\hline
\end{tabular}

\begin{tabular}{|c|c|}
\hline IC348-J & $\begin{array}{c}J \\
{[\mathrm{mag}]}\end{array}$ \\
\hline $034402.3+320923$ & 18.04 \\
\hline $034402.4+321015$ & 14.95 \\
\hline $034402.4+321305$ & 18.92 \\
\hline $034402.4+321102$ & 18.79 \\
\hline $034402.4+321127$ & 16.89 \\
\hline $034402.4+321141$ & 17.20 \\
\hline $034402.4+321720$ & 16.24 \\
\hline $034402.5+321154$ & 18.02 \\
\hline $034402.5+321547$ & 18.37 \\
\hline $034402.5+321523$ & 19.50 \\
\hline $034402.6+320134$ & 12.94 \\
\hline $034402.6+320547$ & 20.26 \\
\hline $034402.6+321415$ & 17.54 \\
\hline $034402.7+321304$ & 19.07 \\
\hline $034402.7+320954$ & 19.90 \\
\hline $034402.9+321528$ & 19.78 \\
\hline $034402.9+320815$ & 19.34 \\
\hline $034402.9+321228$ & 17.00 \\
\hline $034402.9+320615$ & 16.95 \\
\hline $034403.0+320636$ & 19.67 \\
\hline $034403.0+321720$ & 16.83 \\
\hline $034403.0+321619$ & 19.80 \\
\hline $034403.2+320948$ & 18.61 \\
\hline $034403.4+320658$ & 17.82 \\
\hline $034403.5+321523$ & 17.48 \\
\hline $034403.5+321606$ & 20.18 \\
\hline $034403.5+321143$ & 15.32 \\
\hline $034403.6+320232$ & 14.48 \\
\hline $034403.6+320235$ & 14.40 \\
\hline $034403.6+320518$ & 17.55 \\
\hline $034403.6+321639$ & 14.67 \\
\hline $034403.6+320520$ & 15.50 \\
\hline $034403.6+321007$ & 18.05 \\
\hline $034403.7+320548$ & 20.20 \\
\hline $034403.7+320819$ & 18.38 \\
\hline $034403.7+321015$ & 18.07 \\
\hline $034403.8+320648$ & 18.67 \\
\hline $034403.9+321042$ & 18.69 \\
\hline $034403.9+321613$ & 19.34 \\
\hline $034403.9+321637$ & 19.29 \\
\hline $034404.0+321327$ & 19.68 \\
\hline $034404.1+320717$ & 13.03 \\
\hline $034404.2+320949$ & 19.13 \\
\hline $034404.2+321050$ & 19.52 \\
\hline $034404.2+320801$ & 18.38 \\
\hline $034404.2+321406$ & 17.52 \\
\hline $034404.2+320750$ & 19.85 \\
\hline $034404.2+320938$ & 13.65 \\
\hline $034404.2+321349$ & 12.52 \\
\hline $034404.3+321550$ & 14.92 \\
\hline $034404.3+321233$ & 19.48 \\
\hline $034404.4+321555$ & 19.96 \\
\hline $034404.4+321100$ & 19.92 \\
\hline $034404.4+321545$ & 20.21 \\
\hline $034404.4+320453$ & 14.25 \\
\hline $034404.5+321015$ & 20.36 \\
\hline
\end{tabular}


Table 1. continued.

\begin{tabular}{|c|c|}
\hline IC348-J & $\begin{array}{c}J \\
{[\mathrm{mag}]}\end{array}$ \\
\hline $034404.5+321709$ & 20.52 \\
\hline $034404.5+321113$ & 19.61 \\
\hline $034404.6+320838$ & 20.34 \\
\hline $034404.6+321421$ & 18.98 \\
\hline $034404.9+320634$ & 18.50 \\
\hline $034404.9+320650$ & 19.45 \\
\hline $034404.9+320723$ & 19.28 \\
\hline $034404.9+321044$ & 18.86 \\
\hline $034404.9+321656$ & 17.51 \\
\hline $034404.9+320716$ & 19.22 \\
\hline $034405.0+320953$ & 11.52 \\
\hline $034405.0+321538$ & 18.62 \\
\hline $034405.2+320908$ & 20.39 \\
\hline $034405.2+321228$ & 19.38 \\
\hline $034405.2+321406$ & 19.20 \\
\hline $034405.2+320259$ & 19.32 \\
\hline $034405.3+320802$ & 14.23 \\
\hline $034405.3+321311$ & 16.59 \\
\hline $034405.4+320807$ & 18.94 \\
\hline $034405.4+321357$ & 19.57 \\
\hline $034405.5+320900$ & 20.42 \\
\hline $034405.5+321344$ & 17.33 \\
\hline $034405.7+321044$ & 16.06 \\
\hline $034405.8+321229$ & 12.29 \\
\hline $034405.8+321552$ & 19.11 \\
\hline $034405.9+320903$ & 17.73 \\
\hline $034405.9+320639$ & 19.27 \\
\hline $034405.9+321212$ & 18.08 \\
\hline $034405.9+320900$ & 19.06 \\
\hline $034405.9+320420$ & 19.83 \\
\hline $034406.0+321532$ & 14.24 \\
\hline $034406.0+320952$ & 18.47 \\
\hline $034406.0+321059$ & 19.25 \\
\hline $034406.1+321454$ & 18.28 \\
\hline $034406.1+320926$ & 18.14 \\
\hline $034406.1+320707$ & 13.70 \\
\hline $034406.1+321506$ & 16.64 \\
\hline $034406.3+321119$ & 19.52 \\
\hline $034406.3+321035$ & 19.76 \\
\hline $034406.4+321410$ & 18.81 \\
\hline $034406.4+321700$ & 19.94 \\
\hline $034406.5+321441$ & 20.23 \\
\hline $034406.5+321202$ & 16.53 \\
\hline $034406.5+321101$ & 20.08 \\
\hline $034406.6+321640$ & 19.50 \\
\hline $034406.6+321720$ & 16.67 \\
\hline $034406.6+320651$ & 19.42 \\
\hline $034406.6+320741$ & 20.36 \\
\hline $034406.7+320922$ & 20.11 \\
\hline $034406.8+320754$ & 13.03 \\
\hline $034406.8+320440$ & 13.76 \\
\hline $034406.9+321228$ & 20.11 \\
\hline $034406.9+320155$ & 18.31 \\
\hline $034407.0+320826$ & 19.40 \\
\hline $034407.0+321101$ & 18.20 \\
\hline $034407.0+321629$ & 20.58 \\
\hline $034407.1+320844$ & 17.14 \\
\hline $034407.2+321600$ & 21.16 \\
\hline
\end{tabular}

\begin{tabular}{|c|c|}
\hline IC348-J & $\begin{array}{c}J \\
{[\mathrm{mag}]}\end{array}$ \\
\hline $034407.2+321337$ & 19.67 \\
\hline $034407.3+321433$ & 17.76 \\
\hline $034407.3+320948$ & 19.57 \\
\hline $034407.4+321021$ & 18.65 \\
\hline $034407.4+321550$ & 19.67 \\
\hline $034407.4+321655$ & 20.96 \\
\hline $034407.5+320408$ & 13.47 \\
\hline $034407.5+321049$ & 18.92 \\
\hline $034407.6+321330$ & 19.95 \\
\hline $034407.7+320505$ & 14.12 \\
\hline $034407.8+320238$ & 20.18 \\
\hline $034407.8+321409$ & 19.64 \\
\hline $034407.8+321538$ & 19.28 \\
\hline $034407.8+320350$ & 19.39 \\
\hline $034407.8+320635$ & 19.66 \\
\hline $034407.8+321440$ & 16.88 \\
\hline $034407.8+321609$ & 20.93 \\
\hline $034407.9+320754$ & 19.93 \\
\hline $034407.9+321058$ & 19.29 \\
\hline $034408.0+321404$ & 17.93 \\
\hline $034408.0+321244$ & 19.38 \\
\hline $034408.1+320656$ & 13.59 \\
\hline $034408.3+321056$ & 19.10 \\
\hline $034408.3+321445$ & 18.72 \\
\hline $034408.3+321538$ & 15.81 \\
\hline $034408.3+321357$ & 17.69 \\
\hline $034408.4+320927$ & 19.79 \\
\hline $034408.5+320715$ & 8.77 \\
\hline $034408.5+320959$ & 19.24 \\
\hline $034408.5+321613$ & 19.89 \\
\hline $034408.6+321631$ & 19.44 \\
\hline $034408.7+320803$ & 18.17 \\
\hline $034408.8+321521$ & 19.13 \\
\hline $034408.8+320633$ & 19.66 \\
\hline $034408.9+321044$ & 19.17 \\
\hline $034408.9+321610$ & 11.37 \\
\hline $034408.9+320929$ & 20.05 \\
\hline $034409.0+321257$ & 19.32 \\
\hline $034409.0+321329$ & 18.30 \\
\hline $034409.1+321529$ & 18.88 \\
\hline $034409.2+320613$ & 20.21 \\
\hline $034409.2+320708$ & 8.82 \\
\hline $034409.2+321157$ & 20.08 \\
\hline $034409.2+320237$ & 15.68 \\
\hline $034409.4+321110$ & 18.55 \\
\hline $034409.4+321419$ & 16.67 \\
\hline $034409.5+320415$ & 20.28 \\
\hline $034409.7+320738$ & 19.48 \\
\hline $034409.8+321307$ & 17.81 \\
\hline $034409.9+320302$ & 18.05 \\
\hline $034410.0+321123$ & 19.70 \\
\hline $034410.0+320941$ & 14.24 \\
\hline $034410.0+321502$ & 20.13 \\
\hline $034410.0+320804$ & 19.35 \\
\hline $034410.1+320404$ & 13.34 \\
\hline $034410.2+320734$ & 14.70 \\
\hline $034410.3+321400$ & 16.65 \\
\hline $034410.6+321152$ & 18.83 \\
\hline
\end{tabular}

\begin{tabular}{|c|c|}
\hline IC348-J & $\begin{array}{c}J \\
{[\mathrm{mag}]}\end{array}$ \\
\hline $034410.6+321055$ & 17.93 \\
\hline $034410.6+321144$ & 14.98 \\
\hline $034410.6+321431$ & 17.95 \\
\hline $034410.7+321406$ & 19.25 \\
\hline $034410.8+320830$ & 18.93 \\
\hline $034410.8+321215$ & 19.23 \\
\hline $034410.8+321300$ & 16.39 \\
\hline $034410.9+320441$ & 17.62 \\
\hline $034411.0+320315$ & 19.85 \\
\hline $034411.0+320143$ & 16.95 \\
\hline $034411.1+320534$ & 19.58 \\
\hline $034411.2+320816$ & 12.83 \\
\hline $034411.3+320611$ & 12.39 \\
\hline $034411.3+321416$ & 19.66 \\
\hline $034411.3+320906$ & 19.66 \\
\hline $034411.4+320850$ & 18.70 \\
\hline $034411.5+321212$ & 17.71 \\
\hline $034411.6+321424$ & 20.05 \\
\hline $034411.6+320312$ & 12.81 \\
\hline $034411.6+320427$ & 19.07 \\
\hline $034411.7+321217$ & 19.43 \\
\hline $034412.1+320624$ & 19.06 \\
\hline $034412.2+321252$ & 18.14 \\
\hline $034412.2+321134$ & 19.62 \\
\hline $034412.2+321247$ & 19.66 \\
\hline $034412.3+321102$ & 19.82 \\
\hline $034412.3+321221$ & 17.91 \\
\hline $034412.4+321407$ & 17.81 \\
\hline $034412.5+320332$ & 20.49 \\
\hline $034412.8+321055$ & 14.71 \\
\hline $034412.8+321330$ & 20.49 \\
\hline $034412.8+321232$ & 17.74 \\
\hline $034412.9+321324$ & 18.28 \\
\hline $034413.0+320135$ & 15.85 \\
\hline $034413.0+321315$ & 14.37 \\
\hline $034413.0+320435$ & 19.88 \\
\hline $034413.2+320747$ & 19.08 \\
\hline $034413.3+320931$ & 19.97 \\
\hline $034413.3+320444$ & 19.07 \\
\hline $034413.5+320300$ & 15.36 \\
\hline $034413.6+321439$ & 15.30 \\
\hline $034413.7+321408$ & 19.70 \\
\hline $034413.7+320736$ & 19.31 \\
\hline $034413.9+321409$ & 19.01 \\
\hline $034413.9+321027$ & 18.75 \\
\hline $034413.9+320438$ & 19.35 \\
\hline $034414.0+320539$ & 18.14 \\
\hline $034414.1+320354$ & 18.71 \\
\hline $034414.1+321028$ & 12.52 \\
\hline $034414.2+321204$ & 16.34 \\
\hline $034414.2+321403$ & 19.57 \\
\hline $034414.4+321411$ & 20.17 \\
\hline $034414.5+321400$ & 17.93 \\
\hline $034414.5+320403$ & 18.90 \\
\hline $034414.7+321225$ & 19.56 \\
\hline $034414.9+321405$ & 17.78 \\
\hline $034414.9+320612$ & 18.07 \\
\hline $034414.9+321344$ & 18.07 \\
\hline
\end{tabular}


T. Preibisch et al.: IMF and brown dwarf population of IC 348, Online Material p 5

Table 1. continued.

\begin{tabular}{|c|c|}
\hline IC348-J & $\begin{array}{c}J \\
{[\mathrm{mag}]}\end{array}$ \\
\hline $034414.9+320945$ & 18.39 \\
\hline $034415.0+321053$ & 17.62 \\
\hline $034415.0+321120$ & 19.26 \\
\hline $034415.1+320352$ & 18.77 \\
\hline $034415.1+320343$ & 20.10 \\
\hline $034415.1+320427$ & 19.08 \\
\hline $034415.1+320504$ & 20.49 \\
\hline $034415.2+320715$ & 20.16 \\
\hline $034415.4+320603$ & 19.76 \\
\hline $034415.5+320234$ & 12.98 \\
\hline $034415.5+320953$ & 18.56 \\
\hline $034415.6+321014$ & 16.42 \\
\hline $034415.6+321108$ & 19.16 \\
\hline $034415.6+320921$ & 14.60 \\
\hline $034415.6+321445$ & 19.82 \\
\hline $034415.8+321431$ & 14.95 \\
\hline $034415.9+321150$ & 17.01 \\
\hline $034416.0+321209$ & 16.35 \\
\hline $034416.1+320533$ & 17.79 \\
\hline $034416.1+321100$ & 18.83 \\
\hline $034416.2+321118$ & 19.58 \\
\hline $034416.2+320540$ & 17.76 \\
\hline $034416.2+321218$ & 16.87 \\
\hline $034416.2+321345$ & 19.51 \\
\hline $034416.3+321209$ & 18.73 \\
\hline $034416.3+320135$ & 19.81 \\
\hline $034416.3+321157$ & 18.80 \\
\hline $034416.3+321254$ & 17.06 \\
\hline $034416.3+321313$ & 19.35 \\
\hline $034416.4+320955$ & 11.32 \\
\hline $034416.5+321029$ & 17.98 \\
\hline $034416.5+320533$ & 13.39 \\
\hline $034416.6+320525$ & 18.67 \\
\hline $034416.8+321350$ & 18.42 \\
\hline $034417.0+321013$ & 17.45 \\
\hline $034417.1+321317$ & 20.42 \\
\hline $034417.1+320153$ & 18.23 \\
\hline $034417.1+321115$ & 19.32 \\
\hline $034417.2+320520$ & 19.61 \\
\hline $034417.2+320534$ & 20.27 \\
\hline $034417.2+321349$ & 18.40 \\
\hline $034417.3+321107$ & 19.30 \\
\hline $034417.4+321239$ & 20.07 \\
\hline $034417.4+320835$ & 16.74 \\
\hline $034417.6+320529$ & 17.45 \\
\hline $034417.6+321244$ & 20.31 \\
\hline $034417.7+321122$ & 18.04 \\
\hline $034417.7+320136$ & 20.22 \\
\hline $034417.8+320447$ & 13.33 \\
\hline $034417.8+321121$ & 18.04 \\
\hline $034417.8+321040$ & 20.83 \\
\hline $034417.9+321220$ & 12.18 \\
\hline $034417.9+321408$ & 19.71 \\
\hline $034417.9+321435$ & 19.70 \\
\hline $034418.0+320157$ & 18.32 \\
\hline $034418.1+321053$ & 15.50 \\
\hline $034418.1+320833$ & 17.15 \\
\hline
\end{tabular}

\begin{tabular}{|c|c|}
\hline IC348-J & $\begin{array}{c}J \\
{[\mathrm{mag}]}\end{array}$ \\
\hline $034418.2+320456$ & 12.08 \\
\hline $034418.2+321140$ & 15.11 \\
\hline $034418.2+321133$ & 19.98 \\
\hline $034418.2+321422$ & 15.98 \\
\hline $034418.2+320959$ & 13.22 \\
\hline $034418.3+320732$ & 13.78 \\
\hline $034418.3+321112$ & 19.37 \\
\hline $034418.4+320327$ & 19.16 \\
\hline $034418.5+320642$ & 19.99 \\
\hline $034418.6+321253$ & 14.03 \\
\hline $034418.7+321132$ & 18.13 \\
\hline $034418.8+320832$ & 17.58 \\
\hline $034418.9+321133$ & 16.27 \\
\hline $034419.0+320736$ & 14.59 \\
\hline $034419.1+321411$ & 19.71 \\
\hline $034419.2+320930$ & 11.65 \\
\hline $034419.2+321333$ & 19.25 \\
\hline $034419.2+320600$ & 14.46 \\
\hline $034419.2+320848$ & 19.94 \\
\hline $034419.2+320634$ & 19.01 \\
\hline $034419.2+321018$ & 18.54 \\
\hline $034419.2+320734$ & 12.79 \\
\hline $034419.2+321216$ & 19.18 \\
\hline $034419.3+320623$ & 19.96 \\
\hline $034419.3+321248$ & 18.88 \\
\hline $034419.4+321449$ & 19.93 \\
\hline $034419.4+321410$ & 16.34 \\
\hline $034419.4+321430$ & 20.31 \\
\hline $034419.5+321428$ & 19.49 \\
\hline $034419.5+320605$ & 18.60 \\
\hline $034419.6+320224$ & 14.94 \\
\hline $034419.6+320557$ & 18.75 \\
\hline $034419.6+320731$ & 20.51 \\
\hline $034419.7+320645$ & 16.93 \\
\hline $034419.7+320848$ & 19.99 \\
\hline $034419.7+320632$ & 20.23 \\
\hline $034419.8+320324$ & 19.22 \\
\hline $034419.8+321308$ & 18.82 \\
\hline $034419.8+320852$ & 20.66 \\
\hline $034420.0+320848$ & 19.76 \\
\hline $034420.0+320645$ & 13.57 \\
\hline $034420.2+320856$ & 12.67 \\
\hline $034420.3+320544$ & 14.32 \\
\hline $034420.3+320657$ & 20.18 \\
\hline $034420.3+320522$ & 20.50 \\
\hline $034420.4+320320$ & 17.87 \\
\hline $034420.6+320751$ & 19.01 \\
\hline $034420.6+320312$ & 16.70 \\
\hline $034420.7+321015$ & 20.39 \\
\hline $034420.9+321004$ & 18.47 \\
\hline $034420.9+321237$ & 13.94 \\
\hline $034420.9+320858$ & 19.74 \\
\hline $034421.0+320424$ & 18.78 \\
\hline $034421.0+320800$ & 18.70 \\
\hline $034421.0+321335$ & 17.84 \\
\hline $034421.1+320738$ & 11.72 \\
\hline $034421.2+320616$ & 15.28 \\
\hline
\end{tabular}

\begin{tabular}{|c|c|}
\hline IC348-J & $\begin{array}{c}J \\
{[\mathrm{mag}]}\end{array}$ \\
\hline $034421.2+320114$ & 16.52 \\
\hline $034421.3+320502$ & 12.98 \\
\hline $034421.3+321237$ & 13.81 \\
\hline $034421.3+321156$ & 12.68 \\
\hline $034421.4+321121$ & 17.74 \\
\hline $034421.5+320305$ & 19.70 \\
\hline $034421.6+320253$ & 19.31 \\
\hline $034421.6+321017$ & 12.53 \\
\hline $034421.6+321445$ & 18.05 \\
\hline $034421.6+320141$ & 20.28 \\
\hline $034421.6+320508$ & 20.25 \\
\hline $034421.6+321037$ & 12.47 \\
\hline $034421.6+321347$ & 15.83 \\
\hline $034421.7+320624$ & 12.57 \\
\hline $034421.7+321136$ & 19.21 \\
\hline $034421.7+321114$ & 17.08 \\
\hline $034421.8+321231$ & 13.10 \\
\hline $034421.8+321413$ & 19.91 \\
\hline $034421.9+321200$ & 18.63 \\
\hline $034421.9+321211$ & 12.28 \\
\hline $034422.0+321400$ & 17.47 \\
\hline $034422.0+321429$ & 18.96 \\
\hline $034422.1+321327$ & 19.57 \\
\hline $034422.1+321410$ & 18.73 \\
\hline $034422.1+320653$ & 19.83 \\
\hline $034422.2+320333$ & 18.45 \\
\hline $034422.3+320543$ & 12.55 \\
\hline $034422.3+321201$ & 12.52 \\
\hline $034422.4+321301$ & 18.65 \\
\hline $034422.4+321048$ & 20.01 \\
\hline $034422.5+320746$ & 20.41 \\
\hline $034422.6+320127$ & 15.30 \\
\hline $034422.6+320154$ & 12.11 \\
\hline $034422.7+320142$ & 15.27 \\
\hline $034422.8+320205$ & 17.72 \\
\hline $034423.0+320718$ & 18.29 \\
\hline $034423.0+321440$ & 14.17 \\
\hline $034423.0+321157$ & 12.57 \\
\hline $034423.1+321426$ & 18.94 \\
\hline $034423.2+320722$ & 20.62 \\
\hline $034423.3+320717$ & 20.62 \\
\hline $034423.3+320154$ & 15.00 \\
\hline $034423.4+320229$ & 17.81 \\
\hline $034423.5+320814$ & 18.72 \\
\hline $034423.6+320934$ & 13.42 \\
\hline $034423.6+320711$ & 14.48 \\
\hline $034423.6+320153$ & 14.64 \\
\hline $034423.7+320646$ & 12.24 \\
\hline $034423.7+321241$ & 20.23 \\
\hline $034423.8+321156$ & 14.85 \\
\hline $034423.8+321112$ & 20.48 \\
\hline $034423.8+321008$ & 19.46 \\
\hline $034423.9+321421$ & 19.26 \\
\hline $034424.0+320908$ & 20.31 \\
\hline $034424.0+320533$ & 18.97 \\
\hline $034424.0+321059$ & 10.75 \\
\hline $034424.1+321314$ & 17.69 \\
\hline
\end{tabular}


Table 1. continued.

\begin{tabular}{|c|c|}
\hline IC348-J & $\begin{array}{c}J \\
{[\mathrm{mag}]}\end{array}$ \\
\hline $034424.3+320611$ & 17.48 \\
\hline $034424.3+321019$ & 11.40 \\
\hline $034424.3+320758$ & 15.59 \\
\hline $034424.4+320614$ & 20.50 \\
\hline $034424.4+320144$ & 15.74 \\
\hline $034424.5+321305$ & 19.14 \\
\hline $034424.6+320357$ & 12.91 \\
\hline $034424.6+321002$ & 14.05 \\
\hline $034424.6+321140$ & 17.59 \\
\hline $034424.6+321132$ & 19.16 \\
\hline $034424.7+321015$ & 9.37 \\
\hline $034424.8+320543$ & 19.85 \\
\hline $034424.9+321348$ & 17.05 \\
\hline $034424.9+320540$ & 19.87 \\
\hline $034425.0+320446$ & 18.23 \\
\hline $034425.2+320632$ & 20.40 \\
\hline $034425.2+320449$ & 20.25 \\
\hline $034425.2+320536$ & 17.21 \\
\hline $034425.3+321012$ & 12.68 \\
\hline $034425.3+320831$ & 20.16 \\
\hline $034425.4+321316$ & 20.31 \\
\hline $034425.4+320438$ & 19.23 \\
\hline $034425.4+321356$ & 16.86 \\
\hline $034425.4+321405$ & 16.83 \\
\hline $034425.5+320612$ & 20.68 \\
\hline $034425.5+320745$ & 19.37 \\
\hline $034425.5+320617$ & 12.90 \\
\hline $034425.6+320625$ & 15.23 \\
\hline $034425.6+321229$ & 11.85 \\
\hline $034425.6+321130$ & 12.51 \\
\hline $034425.6+321429$ & 18.66 \\
\hline $034425.8+320905$ & 14.56 \\
\hline $034425.8+321058$ & 17.20 \\
\hline $034425.8+320453$ & 20.84 \\
\hline $034425.9+321338$ & 19.92 \\
\hline $034425.9+321106$ & 16.06 \\
\hline $034425.9+320805$ & 19.27 \\
\hline $034426.0+320313$ & 19.21 \\
\hline $034426.0+320430$ & 10.09 \\
\hline $034426.1+320238$ & 19.74 \\
\hline $034426.4+320809$ & 17.87 \\
\hline $034426.4+320827$ & 19.93 \\
\hline $034426.5+321352$ & 18.78 \\
\hline $034426.5+320820$ & 15.43 \\
\hline $034426.6+320358$ & 11.80 \\
\hline $034426.7+320236$ & 14.62 \\
\hline $034426.7+320834$ & 19.68 \\
\hline $034426.7+320820$ & 12.89 \\
\hline $034426.7+320930$ & 20.05 \\
\hline $034426.8+320649$ & 20.13 \\
\hline $034426.8+321218$ & 18.19 \\
\hline $034426.9+320926$ & 16.54 \\
\hline $034426.9+321250$ & 16.33 \\
\hline $034427.0+320443$ & 11.95 \\
\hline $034427.1+320304$ & 20.43 \\
\hline $034427.2+320346$ & 17.29 \\
\hline $034427.2+321006$ & 18.42 \\
\hline $034427.2+321037$ & 13.57 \\
\hline
\end{tabular}

\begin{tabular}{|c|c|}
\hline IC348-J & $\begin{array}{c}J \\
{[\mathrm{mag}]}\end{array}$ \\
\hline $034427.3+320717$ & 14.35 \\
\hline $034427.3+321421$ & 12.77 \\
\hline $034427.3+320755$ & 18.45 \\
\hline $034427.4+321401$ & 16.11 \\
\hline $034427.5+320616$ & 18.99 \\
\hline $034427.5+320218$ & 18.37 \\
\hline $034427.5+320624$ & 20.05 \\
\hline $034427.5+320658$ & 20.47 \\
\hline $034427.5+320225$ & 20.14 \\
\hline $034427.6+320302$ & 15.23 \\
\hline $034427.6+320447$ & 19.10 \\
\hline $034427.6+320843$ & 17.84 \\
\hline $034427.6+320944$ & 18.93 \\
\hline $034427.7+321355$ & 14.82 \\
\hline $034427.7+320851$ & 20.07 \\
\hline $034427.8+320800$ & 17.54 \\
\hline $034427.9+321052$ & 13.82 \\
\hline $034427.9+320731$ & 12.14 \\
\hline $034428.0+320519$ & 14.88 \\
\hline $034428.0+320840$ & 18.75 \\
\hline $034428.2+321029$ & 20.50 \\
\hline $034428.2+321305$ & 17.93 \\
\hline $034428.2+321354$ & 19.34 \\
\hline $034428.2+321219$ & 16.83 \\
\hline $034428.4+321122$ & 14.36 \\
\hline $034428.4+321110$ & 19.69 \\
\hline $034428.5+320722$ & 11.66 \\
\hline $034428.5+321200$ & 18.93 \\
\hline $034428.6+320424$ & 19.24 \\
\hline $034428.6+320441$ & 20.02 \\
\hline $034428.7+320527$ & 20.23 \\
\hline $034428.7+320150$ & 17.95 \\
\hline $034428.7+320207$ & 18.61 \\
\hline $034428.7+320940$ & 19.89 \\
\hline $034428.8+321343$ & 17.16 \\
\hline $034428.8+320423$ & 15.08 \\
\hline $034428.8+321032$ & 19.13 \\
\hline $034428.9+320158$ & 19.91 \\
\hline $034428.9+320702$ & 19.66 \\
\hline $034428.9+320259$ & 20.15 \\
\hline $034428.9+320231$ & 16.17 \\
\hline $034428.9+320138$ & 15.53 \\
\hline $034429.0+321402$ & 17.07 \\
\hline $034429.1+320255$ & 19.49 \\
\hline $034429.1+320751$ & 14.02 \\
\hline $034429.1+320757$ & 13.59 \\
\hline $034429.1+321448$ & 19.11 \\
\hline $034429.2+320115$ & 14.54 \\
\hline $034429.3+321026$ & 18.23 \\
\hline $034429.4+320259$ & 20.22 \\
\hline $034429.5+320404$ & 14.31 \\
\hline $034429.6+320137$ & 18.55 \\
\hline $034429.6+320102$ & 20.36 \\
\hline $034429.6+320729$ & 20.42 \\
\hline $034429.7+321552$ & 18.53 \\
\hline $034429.7+320552$ & 13.61 \\
\hline $034429.7+321039$ & 11.95 \\
\hline $034429.8+320515$ & 19.60 \\
\hline
\end{tabular}

\begin{tabular}{|c|c|}
\hline IC348-J & $\begin{array}{c}J \\
{[\mathrm{mag}]}\end{array}$ \\
\hline $034429.9+320403$ & 19.37 \\
\hline $034430.0+320422$ & 20.23 \\
\hline $034430.0+320921$ & 13.36 \\
\hline $034430.0+320939$ & 15.03 \\
\hline $034430.0+320755$ & 19.37 \\
\hline $034430.0+320849$ & 14.53 \\
\hline $034430.0+321623$ & 20.01 \\
\hline $034430.1+320541$ & 18.14 \\
\hline $034430.1+320118$ & 15.09 \\
\hline $034430.3+320742$ & 13.90 \\
\hline $034430.3+321135$ & 16.57 \\
\hline $034430.3+320944$ & 16.25 \\
\hline $034430.4+320205$ & 20.22 \\
\hline $034430.4+320607$ & 19.31 \\
\hline $034430.4+321310$ & 18.73 \\
\hline $034430.4+321457$ & 15.98 \\
\hline $034430.5+321230$ & 16.96 \\
\hline $034430.6+320629$ & 12.81 \\
\hline $034430.7+320933$ & 19.97 \\
\hline $034430.8+320955$ & 9.84 \\
\hline $034430.9+321251$ & 20.24 \\
\hline $034430.9+321125$ & 17.59 \\
\hline $034431.0+320244$ & 15.63 \\
\hline $034431.0+320558$ & 21.11 \\
\hline $034431.0+320546$ & 14.03 \\
\hline $034431.0+320946$ & 18.93 \\
\hline $034431.1+320146$ & 17.40 \\
\hline $034431.2+320558$ & 15.12 \\
\hline $034431.2+320849$ & 16.53 \\
\hline $034431.2+321327$ & 19.59 \\
\hline $034431.2+320621$ & 8.31 \\
\hline $034431.2+321447$ & 15.24 \\
\hline $034431.3+320928$ & 14.05 \\
\hline $034431.3+320730$ & 16.99 \\
\hline $034431.3+320811$ & 17.34 \\
\hline $034431.4+321047$ & 13.38 \\
\hline $034431.4+320302$ & 19.84 \\
\hline $034431.4+321129$ & 13.52 \\
\hline $034431.5+320706$ & 17.55 \\
\hline $034431.6+320844$ & 10.66 \\
\hline $034431.6+320945$ & 17.69 \\
\hline $034431.6+321454$ & 19.88 \\
\hline $034431.6+321548$ & 19.50 \\
\hline $034431.7+320513$ & 19.62 \\
\hline $034431.7+320653$ & 13.52 \\
\hline $034431.7+320432$ & 19.86 \\
\hline $034431.8+321244$ & 14.65 \\
\hline $034431.8+321400$ & 16.58 \\
\hline $034431.9+321515$ & 19.90 \\
\hline $034431.9+321546$ & 14.19 \\
\hline $034432.0+321144$ & 10.15 \\
\hline $034432.2+320511$ & 19.37 \\
\hline $034432.3+320135$ & 16.25 \\
\hline $034432.3+320729$ & 20.21 \\
\hline $034432.4+320327$ & 14.99 \\
\hline $034432.4+320803$ & 16.07 \\
\hline $034432.6+320842$ & 11.24 \\
\hline $034432.6+320856$ & 12.10 \\
\hline
\end{tabular}


Table 1. continued.

\begin{tabular}{|c|c|}
\hline IC348-J & $\begin{array}{c}J \\
{[\mathrm{mag}]}\end{array}$ \\
\hline $034432.7+320415$ & 13.76 \\
\hline $034432.8+320837$ & 10.28 \\
\hline $034432.8+320915$ & 12.33 \\
\hline $034432.8+320413$ & 13.76 \\
\hline $034432.9+321450$ & 20.15 \\
\hline $034432.9+321518$ & 19.99 \\
\hline $034433.1+321459$ & 14.50 \\
\hline $034433.1+320355$ & 20.18 \\
\hline $034433.2+321528$ & 12.50 \\
\hline $034433.2+320858$ & 19.86 \\
\hline $034433.2+321257$ & 15.17 \\
\hline $034433.3+320752$ & 17.25 \\
\hline $034433.3+320939$ & 12.39 \\
\hline $034433.4+321031$ & 16.58 \\
\hline $034433.5+320316$ & 19.89 \\
\hline $034433.5+321118$ & 19.31 \\
\hline $034433.7+321336$ & 17.51 \\
\hline $034433.7+320521$ & 18.15 \\
\hline $034433.7+320547$ & 17.41 \\
\hline $034433.7+320416$ & 20.05 \\
\hline $034433.8+321321$ & 20.14 \\
\hline $034433.8+321555$ & 19.34 \\
\hline $034433.9+320340$ & 19.91 \\
\hline $034433.9+320730$ & 17.12 \\
\hline $034434.0+321403$ & 17.38 \\
\hline $034434.0+320854$ & 11.83 \\
\hline $034434.0+321541$ & 18.94 \\
\hline $034434.1+320657$ & 14.02 \\
\hline $034434.1+321103$ & 19.36 \\
\hline $034434.2+321635$ & 14.06 \\
\hline $034434.2+320946$ & 6.76 \\
\hline $034434.3+321240$ & 15.35 \\
\hline $034434.3+321448$ & 18.67 \\
\hline $034434.3+321049$ & 12.13 \\
\hline $034434.3+321606$ & 14.34 \\
\hline $034434.4+320422$ & 14.14 \\
\hline $034434.4+321416$ & 18.80 \\
\hline $034434.4+320754$ & 18.35 \\
\hline $034434.5+320625$ & 13.34 \\
\hline $034434.5+320843$ & 16.04 \\
\hline $034434.5+321333$ & 18.44 \\
\hline $034434.6+320213$ & 19.43 \\
\hline $034434.6+320329$ & 19.93 \\
\hline $034434.6+321017$ & 15.89 \\
\hline $034434.7+320339$ & 19.78 \\
\hline $034434.7+321554$ & 13.15 \\
\hline $034434.7+321600$ & 14.98 \\
\hline $034434.7+321516$ & 19.27 \\
\hline $034434.8+320519$ & 19.98 \\
\hline $034434.8+321550$ & 19.62 \\
\hline $034434.8+321117$ & 12.72 \\
\hline $034434.9+320953$ & 12.55 \\
\hline $034434.9+320633$ & 11.50 \\
\hline $034434.9+320604$ & 20.06 \\
\hline $034434.9+321500$ & 16.94 \\
\hline $034434.9+321627$ & 20.24 \\
\hline $034435.0+321530$ & 13.13 \\
\hline $034435.0+320857$ & 14.77 \\
\hline
\end{tabular}

\begin{tabular}{|c|c|}
\hline IC348-J & $\begin{array}{c}J \\
{[\mathrm{mag}]}\end{array}$ \\
\hline $034435.0+320736$ & 10.97 \\
\hline $034435.2+321105$ & 19.82 \\
\hline $034435.3+320332$ & 18.32 \\
\hline $034435.4+320736$ & 12.09 \\
\hline $034435.4+321004$ & 7.92 \\
\hline $034435.4+321104$ & 19.92 \\
\hline $034435.4+320856$ & 13.20 \\
\hline $034435.5+320804$ & 13.56 \\
\hline $034435.5+320212$ & 19.19 \\
\hline $034435.6+320518$ & 19.01 \\
\hline $034435.6+320223$ & 18.78 \\
\hline $034435.7+320248$ & 17.79 \\
\hline $034435.7+320303$ & 14.21 \\
\hline $034435.7+320453$ & 13.79 \\
\hline $034435.8+321101$ & 19.04 \\
\hline $034435.9+321502$ & 13.20 \\
\hline $034435.9+321504$ & 13.79 \\
\hline $034435.9+321553$ & 12.95 \\
\hline $034435.9+321527$ & 19.55 \\
\hline $034435.9+321117$ & 15.72 \\
\hline $034436.0+320924$ & 13.83 \\
\hline $034436.1+320320$ & 19.95 \\
\hline $034436.1+321715$ & 18.85 \\
\hline $034436.1+320717$ & 19.28 \\
\hline $034436.2+321623$ & 17.56 \\
\hline $034436.2+320706$ & 18.60 \\
\hline $034436.3+321304$ & 17.12 \\
\hline $034436.3+320714$ & 20.30 \\
\hline $034436.3+321419$ & 17.23 \\
\hline $034436.3+320513$ & 19.96 \\
\hline $034436.4+320305$ & 16.69 \\
\hline $034436.4+320754$ & 19.99 \\
\hline $034436.4+320919$ & 14.30 \\
\hline $034436.4+321029$ & 16.21 \\
\hline $034436.5+320317$ & 18.56 \\
\hline $034436.6+320903$ & 19.71 \\
\hline $034436.6+320344$ & 16.70 \\
\hline $034436.7+320725$ & 19.15 \\
\hline $034436.7+320901$ & 20.57 \\
\hline $034436.7+321248$ & 19.96 \\
\hline $034436.8+321328$ & 20.42 \\
\hline $034436.9+321418$ & 18.99 \\
\hline $034436.9+321436$ & 15.56 \\
\hline $034437.0+320609$ & 18.71 \\
\hline $034437.0+320645$ & 9.19 \\
\hline $034437.0+320834$ & 12.95 \\
\hline $034437.0+320947$ & 20.18 \\
\hline $034437.0+321656$ & 18.65 \\
\hline $034437.1+320610$ & 19.25 \\
\hline $034437.2+320748$ & 18.14 \\
\hline $034437.2+320915$ & 12.50 \\
\hline $034437.3+321609$ & 20.08 \\
\hline $034437.3+320711$ & 13.90 \\
\hline $034437.3+321255$ & 17.93 \\
\hline $034437.4+320317$ & 20.22 \\
\hline $034437.4+321224$ & 13.08 \\
\hline $034437.4+320611$ & 12.06 \\
\hline $034437.4+320900$ & 12.46 \\
\hline
\end{tabular}

\begin{tabular}{|c|c|}
\hline IC348-J & $\begin{array}{c}J \\
{[\mathrm{mag}]}\end{array}$ \\
\hline $034437.5+321611$ & 19.49 \\
\hline $034437.5+321156$ & 16.77 \\
\hline $034437.6+320344$ & 19.59 \\
\hline $034437.6+321113$ & 19.98 \\
\hline $034437.6+320832$ & 18.89 \\
\hline $034437.8+321217$ & 12.87 \\
\hline $034437.8+320315$ & 19.92 \\
\hline $034437.8+321007$ & 13.95 \\
\hline $034437.9+320508$ & 18.01 \\
\hline $034437.9+320522$ & 17.51 \\
\hline $034437.9+320804$ & 11.66 \\
\hline $034437.9+321500$ & 19.44 \\
\hline $034438.0+320329$ & 11.42 \\
\hline $034438.0+321137$ & 13.22 \\
\hline $034438.1+320433$ & 15.85 \\
\hline $034438.1+321645$ & 15.29 \\
\hline $034438.1+321021$ & 14.27 \\
\hline $034438.1+321031$ & 19.02 \\
\hline $034438.2+321646$ & 16.38 \\
\hline $034438.3+321647$ & 19.26 \\
\hline $034438.3+321047$ & 18.64 \\
\hline $034438.4+321300$ & 12.77 \\
\hline $034438.4+320845$ & 19.51 \\
\hline $034438.5+320735$ & 11.17 \\
\hline $034438.5+320652$ & 18.66 \\
\hline $034438.5+321220$ & 20.04 \\
\hline $034438.5+320800$ & 11.93 \\
\hline $034438.6+321104$ & 18.22 \\
\hline $034438.6+321524$ & 16.72 \\
\hline $034438.6+320506$ & 12.46 \\
\hline $034438.6+321528$ & 19.59 \\
\hline $034438.6+320748$ & 20.05 \\
\hline $034438.7+320856$ & 12.41 \\
\hline $034438.7+320841$ & 11.17 \\
\hline $034438.8+320306$ & 17.73 \\
\hline $034438.8+321447$ & 16.18 \\
\hline $034438.9+320636$ & 13.98 \\
\hline $034438.9+321502$ & 19.86 \\
\hline $034438.9+320608$ & 17.05 \\
\hline $034439.0+320319$ & 14.27 \\
\hline $034439.0+321320$ & 19.96 \\
\hline $034439.0+320514$ & 18.99 \\
\hline $034439.2+320813$ & 14.84 \\
\hline $034439.2+320918$ & 10.05 \\
\hline $034439.2+320945$ & 12.35 \\
\hline $034439.2+320735$ & 10.84 \\
\hline $034439.3+321443$ & 18.06 \\
\hline $034439.3+320928$ & 16.23 \\
\hline $034439.4+321008$ & 13.74 \\
\hline $034439.6+320527$ & 20.41 \\
\hline $034439.8+321345$ & 18.74 \\
\hline $034439.8+321558$ & 13.34 \\
\hline $034439.9+321247$ & 20.16 \\
\hline $034439.9+320613$ & 15.79 \\
\hline $034440.0+320649$ & 20.09 \\
\hline $034440.1+321134$ & 11.66 \\
\hline $034440.1+321427$ & 12.50 \\
\hline $034440.2+320912$ & 13.22 \\
\hline
\end{tabular}


Table 1. continued.

\begin{tabular}{|c|c|}
\hline IC348-J & $\begin{array}{c}J \\
{[\mathrm{mag}]}\end{array}$ \\
\hline $034440.2+321414$ & 16.70 \\
\hline $034440.2+321506$ & 18.38 \\
\hline $034440.2+320932$ & 13.86 \\
\hline $034440.2+321040$ & 19.76 \\
\hline $034440.3+320714$ & 18.91 \\
\hline $034440.3+321426$ & 17.09 \\
\hline $034440.4+320258$ & 20.07 \\
\hline $034440.4+321527$ & 20.22 \\
\hline $034440.5+320304$ & 17.74 \\
\hline $034440.7+320941$ & 15.18 \\
\hline $034440.8+320213$ & 20.14 \\
\hline $034440.8+321306$ & 13.55 \\
\hline $034440.8+320239$ & 19.63 \\
\hline $034440.8+320320$ & 18.68 \\
\hline $034440.8+320455$ & 17.57 \\
\hline $034440.9+321010$ & 13.63 \\
\hline $034440.9+321718$ & 13.80 \\
\hline $034440.9+320615$ & 19.65 \\
\hline $034440.9+321533$ & 18.95 \\
\hline $034441.0+320150$ & 18.11 \\
\hline $034441.0+320623$ & 19.21 \\
\hline $034441.1+320217$ & 15.14 \\
\hline $034441.1+320807$ & 14.53 \\
\hline $034441.1+320346$ & 19.24 \\
\hline $034441.1+321009$ & 13.63 \\
\hline $034441.2+320627$ & 14.27 \\
\hline $034441.2+320412$ & 18.31 \\
\hline $034441.2+320614$ & 18.69 \\
\hline $034441.2+321216$ & 18.74 \\
\hline $034441.3+320936$ & 19.55 \\
\hline $034441.3+320422$ & 16.90 \\
\hline $034441.3+320453$ & 14.54 \\
\hline $034441.3+321025$ & 12.54 \\
\hline $034441.4+321309$ & 13.44 \\
\hline $034441.6+321039$ & 15.36 \\
\hline $034441.6+321313$ & 19.23 \\
\hline $034441.6+320912$ & 20.13 \\
\hline $034441.7+320835$ & 20.01 \\
\hline $034441.7+321202$ & 13.19 \\
\hline $034441.7+320313$ & 18.88 \\
\hline $034441.8+320552$ & 20.39 \\
\hline $034441.8+321417$ & 19.04 \\
\hline $034441.9+320312$ & 19.72 \\
\hline $034441.9+320414$ & 18.68 \\
\hline $034442.0+320446$ & 18.01 \\
\hline $034442.0+320859$ & 12.32 \\
\hline $034442.1+320236$ & 19.18 \\
\hline $034442.1+320258$ & 12.69 \\
\hline $034442.1+320638$ & 20.41 \\
\hline $034442.1+320901$ & 11.86 \\
\hline $034442.3+320744$ & 20.14 \\
\hline $034442.3+321001$ & 20.09 \\
\hline $034442.3+321228$ & 16.13 \\
\hline $034442.3+321401$ & 19.38 \\
\hline $034442.3+321513$ & 16.12 \\
\hline $034442.5+321030$ & 19.77 \\
\hline $034442.6+321002$ & 13.63 \\
\hline $034442.6+320340$ & 19.81 \\
\hline
\end{tabular}

\begin{tabular}{|c|c|}
\hline IC348-J & $\begin{array}{c}J \\
{[\mathrm{mag}]}\end{array}$ \\
\hline $034442.6+321341$ & 18.72 \\
\hline $034442.6+320302$ & 20.36 \\
\hline $034442.6+320619$ & 12.54 \\
\hline $034442.6+321117$ & 19.74 \\
\hline $034442.6+320251$ & 20.36 \\
\hline $034442.6+320700$ & 19.56 \\
\hline $034442.6+321541$ & 15.01 \\
\hline $034442.7+320833$ & 13.12 \\
\hline $034442.9+320535$ & 19.73 \\
\hline $034442.9+321029$ & 17.98 \\
\hline $034442.9+320306$ & 19.59 \\
\hline $034442.9+321318$ & 19.04 \\
\hline $034443.0+320654$ & 14.76 \\
\hline $034443.0+321015$ & 13.38 \\
\hline $034443.0+321559$ & 14.38 \\
\hline $034443.2+320801$ & 19.51 \\
\hline $034443.2+320257$ & 20.15 \\
\hline $034443.2+321239$ & 19.12 \\
\hline $034443.3+320131$ & 17.90 \\
\hline $034443.3+320940$ & 17.32 \\
\hline $034443.4+320817$ & 11.70 \\
\hline $034443.4+321537$ & 19.42 \\
\hline $034443.5+320742$ & 12.14 \\
\hline $034443.5+321212$ & 19.97 \\
\hline $034443.6+321254$ & 20.04 \\
\hline $034443.7+320547$ & 16.40 \\
\hline $034443.7+320906$ & 18.26 \\
\hline $034443.7+321047$ & 14.47 \\
\hline $034443.7+321547$ & 18.81 \\
\hline $034443.8+321523$ & 18.54 \\
\hline $034443.8+320540$ & 19.80 \\
\hline $034443.8+321030$ & 12.29 \\
\hline $034443.8+321351$ & 19.65 \\
\hline $034443.8+321549$ & 19.59 \\
\hline $034443.8+321154$ & 19.04 \\
\hline $034443.8+321539$ & 18.91 \\
\hline $034443.9+320836$ & 17.91 \\
\hline $034444.0+320521$ & 18.55 \\
\hline $034444.0+320539$ & 16.22 \\
\hline $034444.1+320140$ & 20.27 \\
\hline $034444.2+321325$ & 18.89 \\
\hline $034444.2+320943$ & 18.50 \\
\hline $034444.2+320341$ & 17.69 \\
\hline $034444.2+320847$ & 14.50 \\
\hline $034444.3+321036$ & 15.33 \\
\hline $034444.4+320439$ & 20.09 \\
\hline $034444.4+321005$ & 14.97 \\
\hline $034444.5+320514$ & 18.33 \\
\hline $034444.6+320812$ & 12.86 \\
\hline $034444.6+321237$ & 20.15 \\
\hline $034444.6+320730$ & 13.75 \\
\hline $034444.6+320916$ & 20.09 \\
\hline $034444.7+320512$ & 17.20 \\
\hline $034444.7+320402$ & 11.01 \\
\hline $034444.8+321255$ & 18.68 \\
\hline $034444.8+320540$ & 17.21 \\
\hline $034444.8+321028$ & 19.43 \\
\hline $034444.8+321105$ & 13.08 \\
\hline
\end{tabular}

\begin{tabular}{|c|c|}
\hline IC348-J & $\begin{array}{c}J \\
{[\mathrm{mag}]}\end{array}$ \\
\hline $034444.9+320937$ & 18.28 \\
\hline $034444.9+321411$ & 15.81 \\
\hline $034445.0+321336$ & 12.59 \\
\hline $034445.0+320319$ & 19.33 \\
\hline $034445.0+320811$ & 18.23 \\
\hline $034445.1+321413$ & 13.47 \\
\hline $034445.1+320933$ & 19.18 \\
\hline $034445.1+321124$ & 19.67 \\
\hline $034445.1+321356$ & 18.88 \\
\hline $034445.2+320817$ & 18.26 \\
\hline $034445.2+320119$ & 16.21 \\
\hline $034445.2+321055$ & 14.52 \\
\hline $034445.2+320224$ & 18.01 \\
\hline $034445.2+320341$ & 18.00 \\
\hline $034445.4+320556$ & 20.05 \\
\hline $034445.5+320513$ & 17.56 \\
\hline $034445.5+320628$ & 19.14 \\
\hline $034445.5+321403$ & 19.81 \\
\hline $034445.5+320710$ & 17.16 \\
\hline $034445.6+321452$ & 17.91 \\
\hline $034445.6+321110$ & 15.05 \\
\hline $034445.7+321405$ & 19.48 \\
\hline $034445.7+321524$ & 19.90 \\
\hline $034445.8+320624$ & 19.72 \\
\hline $034445.8+320521$ & 19.53 \\
\hline $034445.9+320356$ & 15.28 \\
\hline $034446.0+320353$ & 18.46 \\
\hline $034446.0+321205$ & 15.15 \\
\hline $034446.2+321219$ & 19.34 \\
\hline $034446.2+320810$ & 17.15 \\
\hline $034446.2+320829$ & 19.13 \\
\hline $034446.2+321135$ & 20.42 \\
\hline $034446.2+320312$ & 13.79 \\
\hline $034446.3+320215$ & 17.70 \\
\hline $034446.3+321116$ & 14.00 \\
\hline $034446.3+321342$ & 16.34 \\
\hline $034446.3+321226$ & 20.17 \\
\hline $034446.4+320346$ & 17.80 \\
\hline $034446.4+321331$ & 19.59 \\
\hline $034446.5+321350$ & 18.57 \\
\hline $034446.6+320901$ & 15.33 \\
\hline $034446.7+320323$ & 17.96 \\
\hline $034446.7+320928$ & 20.05 \\
\hline $034446.7+321445$ & 19.94 \\
\hline $034446.8+320616$ & 18.22 \\
\hline $034446.8+320446$ & 19.92 \\
\hline $034446.8+320418$ & 19.31 \\
\hline $034446.8+321153$ & 18.87 \\
\hline $034446.9+320536$ & 14.31 \\
\hline $034446.9+320634$ & 18.29 \\
\hline $034447.3+320527$ & 18.86 \\
\hline $034447.4+320835$ & 18.35 \\
\hline $034447.4+321427$ & 19.56 \\
\hline $034447.4+321516$ & 17.59 \\
\hline $034447.4+320439$ & 20.15 \\
\hline $034447.4+321047$ & 19.67 \\
\hline $034447.5+321123$ & 20.10 \\
\hline $034447.6+320446$ & 19.84 \\
\hline
\end{tabular}


Table 1. continued.

\begin{tabular}{|c|c|}
\hline IC348-J & $\begin{array}{c}J \\
{[\mathrm{mag}]}\end{array}$ \\
\hline $034447.6+321714$ & 16.46 \\
\hline $034447.6+321055$ & 13.51 \\
\hline $034447.7+320353$ & 17.64 \\
\hline $034447.7+321255$ & 19.72 \\
\hline $034447.9+321325$ & 20.06 \\
\hline $034447.9+320256$ & 20.32 \\
\hline $034447.9+320404$ & 19.15 \\
\hline $034447.9+320951$ & 18.80 \\
\hline $034448.0+321140$ & 18.95 \\
\hline $034448.1+320737$ & 19.95 \\
\hline $034448.1+321500$ & 18.68 \\
\hline $034448.1+321522$ & 17.02 \\
\hline $034448.1+321627$ & 20.17 \\
\hline $034448.3+321226$ & 17.57 \\
\hline $034448.4+320711$ & 20.31 \\
\hline $034448.4+320859$ & 18.73 \\
\hline $034448.5+321527$ & 11.40 \\
\hline $034448.5+321518$ & 20.53 \\
\hline $034448.6+320530$ & 20.02 \\
\hline $034448.7+321703$ & 20.18 \\
\hline $034448.7+320140$ & 19.59 \\
\hline $034448.8+321322$ & 13.24 \\
\hline $034448.8+320419$ & 17.11 \\
\hline $034448.9+320733$ & 20.29 \\
\hline $034448.9+320407$ & 19.33 \\
\hline $034449.0+321626$ & 19.58 \\
\hline $034449.1+320847$ & 19.83 \\
\hline $034449.1+320505$ & 20.18 \\
\hline $034449.3+320949$ & 18.46 \\
\hline $034449.4+321504$ & 18.68 \\
\hline $034449.5+321020$ & 16.33 \\
\hline $034449.5+321217$ & 16.53 \\
\hline $034449.6+320911$ & 13.22 \\
\hline $034449.6+321129$ & 18.98 \\
\hline $034449.6+321645$ & 11.17 \\
\hline $034449.7+321407$ & 19.64 \\
\hline $034449.8+320941$ & 19.00 \\
\hline $034449.8+320334$ & 13.18 \\
\hline $034449.8+321731$ & 20.45 \\
\hline $034449.8+320844$ & 18.66 \\
\hline $034449.9+321608$ & 18.81 \\
\hline $034449.9+320614$ & 18.01 \\
\hline $034449.9+321706$ & 18.06 \\
\hline $034449.9+320841$ & 17.19 \\
\hline $034449.9+321602$ & 17.09 \\
\hline $034450.0+320345$ & 12.51 \\
\hline $034450.0+321117$ & 15.31 \\
\hline $034450.1+320747$ & 19.26 \\
\hline $034450.1+320843$ & 17.70 \\
\hline $034450.2+320635$ & 18.86 \\
\hline $034450.2+320714$ & 18.17 \\
\hline $034450.3+321351$ & 18.78 \\
\hline $034450.3+321635$ & 19.17 \\
\hline $034450.3+320345$ & 19.13 \\
\hline $034450.4+320356$ & 20.53 \\
\hline $034450.4+320515$ & 20.12 \\
\hline $034450.4+320754$ & 19.16 \\
\hline $034450.5+321209$ & 18.02 \\
\hline
\end{tabular}

\begin{tabular}{|c|c|}
\hline IC348-J & $\begin{array}{c}J \\
{[\mathrm{mag}]}\end{array}$ \\
\hline $034450.5+320800$ & 20.24 \\
\hline $034450.6+320615$ & 19.48 \\
\hline $034450.7+320521$ & 19.66 \\
\hline $034450.7+321718$ & 15.89 \\
\hline $034450.8+321414$ & 18.32 \\
\hline $034450.9+321629$ & 17.87 \\
\hline $034451.0+321609$ & 12.36 \\
\hline $034451.1+320649$ & 20.13 \\
\hline $034451.2+320359$ & 19.18 \\
\hline $034451.2+321327$ & 19.90 \\
\hline $034451.2+321504$ & 14.05 \\
\hline $034451.4+320946$ & 17.22 \\
\hline $034451.5+320751$ & 18.76 \\
\hline $034451.5+320429$ & 16.92 \\
\hline $034451.6+321213$ & 20.50 \\
\hline $034451.7+321402$ & 20.42 \\
\hline $034451.8+320346$ & 19.96 \\
\hline $034451.8+320917$ & 18.42 \\
\hline $034451.8+320950$ & 15.60 \\
\hline $034451.8+321118$ & 17.55 \\
\hline $034451.8+320835$ & 19.63 \\
\hline $034451.9+321336$ & 20.17 \\
\hline $034451.9+320521$ & 20.31 \\
\hline $034451.9+320628$ & 18.37 \\
\hline $034451.9+320957$ & 17.88 \\
\hline $034451.9+321319$ & 18.18 \\
\hline $034451.9+321635$ & 15.27 \\
\hline $034452.0+320617$ & 20.27 \\
\hline $034452.1+320446$ & 13.89 \\
\hline $034452.1+321006$ & 19.48 \\
\hline $034452.1+321028$ & 18.37 \\
\hline $034452.2+320512$ & 19.24 \\
\hline $034452.3+321022$ & 19.59 \\
\hline $034452.3+320717$ & 20.02 \\
\hline $034452.3+321127$ & 17.41 \\
\hline $034452.3+321455$ & 20.07 \\
\hline $034452.4+321337$ & 19.32 \\
\hline $034452.5+320710$ & 17.17 \\
\hline $034452.5+320741$ & 16.28 \\
\hline $034452.5+321634$ & 19.03 \\
\hline $034452.6+321006$ & 19.06 \\
\hline $034452.6+321008$ & 17.68 \\
\hline $034452.6+321032$ & 19.31 \\
\hline $034452.6+321331$ & 19.97 \\
\hline $034452.7+320202$ & 20.19 \\
\hline $034452.7+320654$ & 20.10 \\
\hline $034452.7+321703$ & 19.70 \\
\hline $034452.7+321708$ & 19.45 \\
\hline $034452.7+321017$ & 17.13 \\
\hline $034452.9+320942$ & 17.75 \\
\hline $034452.9+321705$ & 18.14 \\
\hline $034453.0+320507$ & 20.32 \\
\hline $034453.0+320655$ & 19.64 \\
\hline $034453.1+320518$ & 15.91 \\
\hline $034453.1+321219$ & 20.22 \\
\hline $034453.1+321311$ & 19.83 \\
\hline $034453.3+320430$ & 18.99 \\
\hline $034453.3+321133$ & 19.87 \\
\hline
\end{tabular}

\begin{tabular}{|c|c|}
\hline IC348-J & $\begin{array}{c}J \\
{[\mathrm{mag}]}\end{array}$ \\
\hline $034453.4+321110$ & 18.92 \\
\hline $034453.4+321539$ & 20.31 \\
\hline $034453.4+321550$ & 18.83 \\
\hline $034453.5+320617$ & 18.37 \\
\hline $034453.5+321652$ & 19.55 \\
\hline $034453.5+321356$ & 17.57 \\
\hline $034453.6+321404$ & 19.97 \\
\hline $034453.6+320343$ & 17.72 \\
\hline $034453.6+320858$ & 14.83 \\
\hline $034453.6+321557$ & 20.03 \\
\hline $034453.6+321721$ & 20.49 \\
\hline $034453.7+320652$ & 13.19 \\
\hline $034453.8+321118$ & 19.61 \\
\hline $034453.9+320615$ & 20.13 \\
\hline $034453.9+320436$ & 20.37 \\
\hline $034453.9+321024$ & 19.45 \\
\hline $034453.9+321045$ & 20.22 \\
\hline $034453.9+320913$ & 19.20 \\
\hline $034453.9+321349$ & 18.83 \\
\hline $034453.9+321508$ & 18.86 \\
\hline $034454.0+321723$ & 18.52 \\
\hline $034454.0+320230$ & 19.28 \\
\hline $034454.0+320931$ & 19.77 \\
\hline $034454.0+321524$ & 19.04 \\
\hline $034454.1+321653$ & 20.03 \\
\hline $034454.2+320427$ & 18.05 \\
\hline $034454.2+321117$ & 19.94 \\
\hline $034454.4+320613$ & 18.93 \\
\hline $034454.5+321015$ & 18.95 \\
\hline $034454.7+320440$ & 19.35 \\
\hline $034454.7+320611$ & 20.17 \\
\hline $034454.9+321345$ & 19.94 \\
\hline $034455.0+321210$ & 10.43 \\
\hline $034455.0+321447$ & 19.87 \\
\hline $034455.0+321006$ & 16.98 \\
\hline $034455.1+320557$ & 19.98 \\
\hline $034455.1+321154$ & 17.90 \\
\hline $034455.1+321438$ & 19.38 \\
\hline $034455.2+320151$ & 19.79 \\
\hline $034455.2+321008$ & 20.46 \\
\hline $034455.2+321213$ & 14.32 \\
\hline $034455.2+321503$ & 18.11 \\
\hline $034455.2+321520$ & 18.91 \\
\hline $034455.2+320656$ & 19.86 \\
\hline $034455.2+321506$ & 18.50 \\
\hline $034455.3+320541$ & 17.89 \\
\hline $034455.3+320912$ & 18.25 \\
\hline $034455.3+321502$ & 14.78 \\
\hline $034455.3+320729$ & 16.32 \\
\hline $034455.3+320934$ & 13.47 \\
\hline $034455.4+320516$ & 15.47 \\
\hline $034455.4+321351$ & 16.92 \\
\hline $034455.5+320524$ & 18.38 \\
\hline $034455.5+321414$ & 16.88 \\
\hline $034455.5+320123$ & 20.12 \\
\hline $034455.5+321307$ & 17.12 \\
\hline $034455.5+321346$ & 19.06 \\
\hline $034455.6+320919$ & 11.87 \\
\hline
\end{tabular}


Table 1. continued.

\begin{tabular}{|c|c|}
\hline IC348-J & $\begin{array}{c}J \\
{[\mathrm{mag}]}\end{array}$ \\
\hline $034455.7+321032$ & 19.51 \\
\hline $034455.7+320619$ & 19.95 \\
\hline $034455.8+320405$ & 20.20 \\
\hline $034455.8+321403$ & 17.41 \\
\hline $034455.8+320844$ & 15.62 \\
\hline $034455.8+321313$ & 17.66 \\
\hline $034455.9+321137$ & 20.14 \\
\hline $034455.9+320726$ & 13.67 \\
\hline $034456.0+321001$ & 19.40 \\
\hline $034456.0+321351$ & 15.28 \\
\hline $034456.1+320355$ & 17.29 \\
\hline $034456.1+320556$ & 12.97 \\
\hline $034456.1+320504$ & 19.38 \\
\hline $034456.1+320534$ & 16.37 \\
\hline $034456.1+320915$ & 11.03 \\
\hline $034456.2+320901$ & 20.20 \\
\hline $034456.2+321048$ & 19.58 \\
\hline $034456.3+320933$ & 20.34 \\
\hline $034456.3+321031$ & 15.87 \\
\hline $034456.3+321035$ & 16.58 \\
\hline $034456.4+320729$ & 19.93 \\
\hline $034456.4+321438$ & 18.72 \\
\hline $034456.4+321700$ & 16.71 \\
\hline $034456.4+321335$ & 18.52 \\
\hline $034456.4+320310$ & 19.66 \\
\hline $034456.5+320207$ & 19.48 \\
\hline $034456.5+320444$ & 19.81 \\
\hline $034456.5+320634$ & 19.73 \\
\hline $034456.6+321131$ & 20.10 \\
\hline $034456.7+321225$ & 17.69 \\
\hline $034456.8+321134$ & 19.91 \\
\hline $034456.8+321703$ & 16.56 \\
\hline $034456.8+320324$ & 19.56 \\
\hline $034456.8+320545$ & 13.58 \\
\hline $034456.9+320914$ & 16.34 \\
\hline $034456.9+320418$ & 19.66 \\
\hline $034457.0+320346$ & 19.68 \\
\hline $034457.1+320211$ & 19.34 \\
\hline $034457.1+321348$ & 15.50 \\
\hline $034457.2+321225$ & 20.21 \\
\hline $034457.2+320234$ & 18.38 \\
\hline $034457.2+321007$ & 19.43 \\
\hline $034457.3+320850$ & 18.31 \\
\hline $034457.3+320835$ & 17.82 \\
\hline $034457.4+320115$ & 19.91 \\
\hline $034457.4+320211$ & 20.15 \\
\hline $034457.4+320258$ & 17.57 \\
\hline $034457.5+320942$ & 18.20 \\
\hline $034457.5+321521$ & 18.38 \\
\hline $034457.5+320809$ & 19.85 \\
\hline $034457.6+320631$ & 18.17 \\
\hline $034457.6+321718$ & 18.17 \\
\hline $034457.6+320701$ & 13.55 \\
\hline $034457.6+321224$ & 12.31 \\
\hline $034457.7+320741$ & 14.16 \\
\hline $034457.7+320744$ & 21.68 \\
\hline $034457.7+320837$ & 18.40 \\
\hline $034457.8+320722$ & 17.17 \\
\hline
\end{tabular}

\begin{tabular}{|c|c|}
\hline IC348-J & $\begin{array}{c}J \\
{[\mathrm{mag}]}\end{array}$ \\
\hline $034457.8+321100$ & 20.11 \\
\hline $034457.9+320401$ & 13.45 \\
\hline $034457.9+320716$ & 19.24 \\
\hline $034458.0+321155$ & 18.81 \\
\hline $034458.0+321153$ & 20.41 \\
\hline $034458.0+320454$ & 18.66 \\
\hline $034458.1+321250$ & 17.65 \\
\hline $034458.2+320710$ & 15.39 \\
\hline $034458.2+321314$ & 18.56 \\
\hline $034458.2+320408$ & 17.23 \\
\hline $034458.2+321527$ & 18.13 \\
\hline $034458.3+321633$ & 19.57 \\
\hline $034458.4+320844$ & 18.83 \\
\hline $034458.4+320207$ & 16.61 \\
\hline $034458.6+320710$ & 18.67 \\
\hline $034458.8+320638$ & 18.61 \\
\hline $034458.8+320323$ & 20.05 \\
\hline $034458.8+321529$ & 20.21 \\
\hline $034459.0+320654$ & 20.32 \\
\hline $034459.0+320901$ & 17.60 \\
\hline $034459.0+320441$ & 18.76 \\
\hline $034459.0+320433$ & 19.26 \\
\hline $034459.0+321455$ & 17.27 \\
\hline $034459.1+320437$ & 20.37 \\
\hline $034459.1+321010$ & 16.34 \\
\hline $034459.1+321102$ & 17.41 \\
\hline $034459.1+321422$ & 16.44 \\
\hline $034459.2+320253$ & 18.33 \\
\hline $034459.3+320605$ & 19.59 \\
\hline $034459.3+320634$ & 20.08 \\
\hline $034459.4+320139$ & 19.80 \\
\hline $034459.4+320141$ & 17.62 \\
\hline $034459.4+320907$ & 17.80 \\
\hline $034459.4+320437$ & 18.29 \\
\hline $034459.4+321338$ & 20.12 \\
\hline $034459.5+321039$ & 13.25 \\
\hline $034459.6+321424$ & 14.31 \\
\hline $034459.6+320633$ & 18.16 \\
\hline $034459.7+321131$ & 19.87 \\
\hline $034459.8+320647$ & 14.62 \\
\hline $034459.9+321332$ & 13.76 \\
\hline $034459.9+320802$ & 20.22 \\
\hline $034459.9+320647$ & 14.62 \\
\hline $034459.9+320242$ & 20.02 \\
\hline $034500.1+321302$ & 17.18 \\
\hline $034500.1+320430$ & 19.09 \\
\hline $034500.2+320651$ & 18.38 \\
\hline $034500.2+321324$ & 14.56 \\
\hline $034500.3+321251$ & 18.97 \\
\hline $034500.4+321248$ & 14.32 \\
\hline $034500.5+320320$ & 15.48 \\
\hline $034500.5+321047$ & 18.50 \\
\hline $034500.5+321258$ & 18.80 \\
\hline $034500.5+320534$ & 19.39 \\
\hline $034500.5+320616$ & 18.62 \\
\hline $034500.5+321403$ & 18.06 \\
\hline $034500.6+320819$ & 14.21 \\
\hline $034500.7+321057$ & 18.35 \\
\hline
\end{tabular}

\begin{tabular}{|c|c|}
\hline IC348-J & $\begin{array}{c}J \\
{[\mathrm{mag}]}\end{array}$ \\
\hline $034500.8+321229$ & 17.20 \\
\hline $034500.8+321635$ & 17.56 \\
\hline $034500.9+320808$ & 16.82 \\
\hline $034500.9+320352$ & 19.58 \\
\hline $034501.0+320953$ & 18.52 \\
\hline $034501.0+320955$ & 19.25 \\
\hline $034501.0+321222$ & 14.20 \\
\hline $034501.0+321605$ & 20.32 \\
\hline $034501.1+321426$ & 20.19 \\
\hline $034501.1+320320$ & 13.96 \\
\hline $034501.1+320226$ & 14.35 \\
\hline $034501.2+321031$ & 19.90 \\
\hline $034501.3+320938$ & 18.93 \\
\hline $034501.3+320420$ & 20.07 \\
\hline $034501.3+321313$ & 15.47 \\
\hline $034501.4+321002$ & 20.13 \\
\hline $034501.5+320500$ & 10.32 \\
\hline $034501.5+320239$ & 19.86 \\
\hline $034501.5+321229$ & 13.26 \\
\hline $034501.5+321315$ & 18.19 \\
\hline $034501.5+321446$ & 19.17 \\
\hline $034501.5+321051$ & 11.89 \\
\hline $034501.6+321109$ & 17.54 \\
\hline $034501.6+321317$ & 14.43 \\
\hline $034501.6+320935$ & 18.09 \\
\hline $034501.7+320429$ & 17.80 \\
\hline $034501.7+320254$ & 19.55 \\
\hline $034501.8+321210$ & 19.30 \\
\hline $034501.8+321427$ & 11.36 \\
\hline $034501.8+321434$ & 17.60 \\
\hline $034501.8+320650$ & 17.83 \\
\hline $034501.8+320242$ & 19.25 \\
\hline $034502.0+320744$ & 20.10 \\
\hline $034502.1+321455$ & 17.67 \\
\hline $034502.1+321355$ & 18.46 \\
\hline $034502.3+320628$ & 20.03 \\
\hline $034502.3+321115$ & 20.20 \\
\hline $034502.3+320816$ & 17.74 \\
\hline $034502.3+321031$ & 19.83 \\
\hline $034502.4+320614$ & 20.00 \\
\hline $034502.4+321016$ & 18.43 \\
\hline $034502.5+320929$ & 19.27 \\
\hline $034502.5+321213$ & 19.29 \\
\hline $034502.6+320728$ & 19.46 \\
\hline $034502.6+321046$ & 18.86 \\
\hline $034502.6+321331$ & 16.60 \\
\hline $034502.6+320633$ & 16.39 \\
\hline $034502.7+321248$ & 19.57 \\
\hline $034502.7+321524$ & 16.93 \\
\hline $034502.7+321111$ & 18.52 \\
\hline $034502.8+320544$ & 17.87 \\
\hline $034502.8+320700$ & 12.69 \\
\hline $034502.8+321407$ & 17.03 \\
\hline $034502.9+320240$ & 17.98 \\
\hline $034502.9+320550$ & 16.78 \\
\hline $034502.9+321127$ & 19.31 \\
\hline $034502.9+321435$ & 20.30 \\
\hline $034503.0+320444$ & 19.97 \\
\hline
\end{tabular}


Table 1. continued.

\begin{tabular}{|c|c|}
\hline IC348-J & $\begin{array}{c}J \\
{[\mathrm{mag}]}\end{array}$ \\
\hline $034503.0+320122$ & 17.73 \\
\hline $034503.0+321125$ & 15.97 \\
\hline $034503.1+320617$ & 15.87 \\
\hline $034503.1+321252$ & 17.85 \\
\hline $034503.2+321421$ & 19.93 \\
\hline $034503.2+321150$ & 18.16 \\
\hline $034503.2+320405$ & 11.94 \\
\hline $034503.2+320714$ & 17.17 \\
\hline $034503.2+321529$ & 18.57 \\
\hline $034503.3+320706$ & 20.41 \\
\hline $034503.3+321311$ & 20.35 \\
\hline $034503.3+321417$ & 18.26 \\
\hline $034503.4+321549$ & 20.24 \\
\hline $034503.5+320858$ & 18.96 \\
\hline $034503.6+321435$ & 19.72 \\
\hline $034503.6+321213$ & 17.25 \\
\hline $034503.7+320659$ & 19.83 \\
\hline $034503.7+321043$ & 18.83 \\
\hline $034503.8+321320$ & 19.47 \\
\hline $034503.8+320237$ & 17.77 \\
\hline $034503.9+320812$ & 17.81 \\
\hline $034504.0+320251$ & 19.85 \\
\hline $034504.0+320656$ & 19.46 \\
\hline $034504.0+321007$ & 19.67 \\
\hline $034504.1+320259$ & 20.43 \\
\hline $034504.1+320540$ & 19.92 \\
\hline $034504.1+320504$ & 16.45 \\
\hline $034504.1+320813$ & 18.28 \\
\hline $034504.1+321104$ & 17.24 \\
\hline $034504.2+320603$ & 18.20 \\
\hline $034504.2+320739$ & 18.72 \\
\hline $034504.2+320746$ & 16.83 \\
\hline $034504.2+320559$ & 16.50 \\
\hline $034504.2+320332$ & 19.71 \\
\hline $034504.3+320844$ & 19.87 \\
\hline $034504.3+321324$ & 18.06 \\
\hline $034504.3+320305$ & 14.06 \\
\hline $034504.4+321522$ & 20.36 \\
\hline $034504.5+321027$ & 19.67 \\
\hline $034504.5+320306$ & 16.95 \\
\hline $034504.5+320133$ & 17.01 \\
\hline $034504.5+321425$ & 19.95 \\
\hline $034504.6+320830$ & 19.39 \\
\hline $034504.6+320504$ & 19.25 \\
\hline $034504.7+321501$ & 13.42 \\
\hline $034504.7+321638$ & 13.41 \\
\hline $034504.8+320400$ & 19.77 \\
\hline $034504.9+320211$ & 19.99 \\
\hline $034504.9+321219$ & 19.83 \\
\hline $034504.9+321607$ & 19.03 \\
\hline $034504.9+321018$ & 19.28 \\
\hline $034504.9+321722$ & 19.60 \\
\hline $034504.9+320732$ & 16.86 \\
\hline $034505.0+321655$ & 20.08 \\
\hline $034505.0+321442$ & 18.41 \\
\hline $034505.1+321132$ & 19.51 \\
\hline $034505.1+321655$ & 17.17 \\
\hline $034505.1+321635$ & 19.75 \\
\hline
\end{tabular}

\begin{tabular}{|c|c|}
\hline IC348-J & $\begin{array}{c}J \\
{[\mathrm{mag}]}\end{array}$ \\
\hline $034505.2+321628$ & 17.25 \\
\hline $034505.2+320954$ & 12.99 \\
\hline $034505.4+321216$ & 15.32 \\
\hline $034505.4+321227$ & 19.70 \\
\hline $034505.4+320420$ & 17.61 \\
\hline $034505.4+320308$ & 14.38 \\
\hline $034505.4+320920$ & 20.46 \\
\hline $034505.6+321130$ & 19.12 \\
\hline $034505.7+321417$ & 15.24 \\
\hline $034505.8+320308$ & 11.76 \\
\hline $034505.9+320119$ & 18.89 \\
\hline $034505.9+320826$ & 16.03 \\
\hline $034505.9+320238$ & 17.24 \\
\hline $034505.9+321426$ & 19.89 \\
\hline $034506.0+321216$ & 17.65 \\
\hline $034506.0+321555$ & 19.68 \\
\hline $034506.2+321556$ & 18.54 \\
\hline $034506.2+321304$ & 18.50 \\
\hline $034506.2+321400$ & 19.04 \\
\hline $034506.3+320552$ & 17.22 \\
\hline $034506.4+320709$ & 16.03 \\
\hline $034506.4+321704$ & 18.51 \\
\hline $034506.6+321019$ & 18.12 \\
\hline $034506.6+321533$ & 19.16 \\
\hline $034506.7+320834$ & 17.32 \\
\hline $034506.7+320930$ & 13.71 \\
\hline $034506.7+320535$ & 16.07 \\
\hline $034506.8+320755$ & 18.16 \\
\hline $034506.8+321253$ & 18.48 \\
\hline $034506.8+321350$ & 19.75 \\
\hline $034506.8+320539$ & 18.51 \\
\hline $034506.8+320541$ & 16.95 \\
\hline $034506.9+321242$ & 15.10 \\
\hline $034506.9+321614$ & 20.12 \\
\hline $034506.9+321352$ & 17.15 \\
\hline $034506.9+321506$ & 18.34 \\
\hline $034507.0+321702$ & 18.69 \\
\hline $034507.0+321245$ & 20.48 \\
\hline $034507.0+321403$ & 16.40 \\
\hline $034507.0+320232$ & 19.27 \\
\hline $034507.0+320607$ & 19.61 \\
\hline $034507.1+321530$ & 19.91 \\
\hline $034507.2+320651$ & 16.58 \\
\hline $034507.2+320748$ & 20.08 \\
\hline $034507.2+321310$ & 19.86 \\
\hline $034507.4+320242$ & 19.56 \\
\hline $034507.4+320712$ & 20.28 \\
\hline $034507.5+320909$ & 14.63 \\
\hline $034507.5+321435$ & 18.35 \\
\hline $034507.5+320855$ & 17.43 \\
\hline $034507.5+320952$ & 19.80 \\
\hline $034507.6+320514$ & 16.35 \\
\hline $034507.6+320222$ & 19.49 \\
\hline $034507.6+321027$ & 10.14 \\
\hline $034507.6+321040$ & 19.69 \\
\hline $034507.7+320818$ & 19.51 \\
\hline $034507.7+320338$ & 14.07 \\
\hline $034507.7+320836$ & 18.33 \\
\hline
\end{tabular}

\begin{tabular}{|c|c|}
\hline IC348-J & $\begin{array}{c}J \\
{[\mathrm{mag}]}\end{array}$ \\
\hline $034507.9+321639$ & 19.22 \\
\hline $034507.9+320532$ & 19.34 \\
\hline $034508.0+321637$ & 20.23 \\
\hline $034508.0+321551$ & 19.74 \\
\hline $034508.0+320354$ & 19.29 \\
\hline $034508.0+320400$ & 10.28 \\
\hline $034508.1+320412$ & 18.71 \\
\hline $034508.3+320429$ & 18.91 \\
\hline $034508.3+320915$ & 17.89 \\
\hline $034508.3+321307$ & 19.15 \\
\hline $034508.3+321523$ & 20.09 \\
\hline $034508.3+321336$ & 18.17 \\
\hline $034508.3+321004$ & 19.79 \\
\hline $034508.4+320701$ & 16.16 \\
\hline $034508.5+321149$ & 20.06 \\
\hline $034508.5+321031$ & 18.47 \\
\hline $034508.6+320234$ & 17.54 \\
\hline $034508.6+320606$ & 20.05 \\
\hline $034508.7+320924$ & 20.13 \\
\hline $034508.8+321357$ & 19.27 \\
\hline $034508.9+320244$ & 20.09 \\
\hline $034508.9+321546$ & 18.17 \\
\hline $034508.9+321147$ & 19.83 \\
\hline $034509.0+320547$ & 19.56 \\
\hline $034509.2+320114$ & 16.26 \\
\hline $034509.2+321615$ & 19.83 \\
\hline $034509.3+320805$ & 20.24 \\
\hline $034509.3+321221$ & 20.16 \\
\hline $034509.3+321519$ & 16.19 \\
\hline $034509.5+321303$ & 15.98 \\
\hline $034509.5+321111$ & 18.59 \\
\hline $034509.6+320902$ & 20.09 \\
\hline $034509.6+321302$ & 17.87 \\
\hline $034509.6+320828$ & 18.55 \\
\hline $034509.6+321338$ & 13.98 \\
\hline $034509.6+321603$ & 11.95 \\
\hline $034509.7+320158$ & 19.47 \\
\hline $034509.9+321335$ & 14.13 \\
\hline $034509.9+321525$ & 19.84 \\
\hline $034510.0+320533$ & 19.66 \\
\hline $034510.1+320449$ & 14.91 \\
\hline $034510.1+320945$ & 17.38 \\
\hline $034510.2+320919$ & 20.03 \\
\hline $034510.2+321304$ & 16.97 \\
\hline $034510.2+321457$ & 20.16 \\
\hline $034510.3+320858$ & 17.83 \\
\hline $034510.6+321427$ & 17.95 \\
\hline $034510.7+321318$ & 18.60 \\
\hline $034510.7+321410$ & 20.17 \\
\hline $034510.8+321300$ & 20.21 \\
\hline $034510.8+320330$ & 20.20 \\
\hline $034510.8+321626$ & 17.97 \\
\hline $034510.8+321305$ & 18.91 \\
\hline $034510.9+320753$ & 18.84 \\
\hline $034510.9+320743$ & 18.11 \\
\hline $034510.9+321340$ & 18.50 \\
\hline $034511.0+321700$ & 15.79 \\
\hline $034511.1+320852$ & 14.33 \\
\hline
\end{tabular}


Table 1. continued.

\begin{tabular}{|c|c|}
\hline IC348-J & $\begin{array}{c}J \\
{[\mathrm{mag}]}\end{array}$ \\
\hline $034511.2+321637$ & 17.43 \\
\hline $034511.2+320749$ & 19.59 \\
\hline $034511.3+321453$ & 19.47 \\
\hline $034511.3+321655$ & 20.16 \\
\hline $034511.3+321346$ & 18.46 \\
\hline $034511.4+320335$ & 18.73 \\
\hline $034511.4+320141$ & 19.01 \\
\hline $034511.4+320811$ & 20.16 \\
\hline $034511.5+321424$ & 20.13 \\
\hline $034511.6+321103$ & 17.90 \\
\hline $034511.7+321305$ & 18.64 \\
\hline $034511.8+320126$ & 17.83 \\
\hline $034511.9+321056$ & 19.41 \\
\hline $034511.9+321124$ & 18.07 \\
\hline $034512.0+320256$ & 19.98 \\
\hline $034512.0+321042$ & 17.85 \\
\hline $034512.1+321141$ & 13.62 \\
\hline $034512.1+320913$ & 14.00 \\
\hline $034512.1+321029$ & 12.62 \\
\hline $034512.2+320550$ & 18.40 \\
\hline $034512.2+321200$ & 14.50 \\
\hline $034512.2+321352$ & 17.19 \\
\hline $034512.4+321440$ & 19.50 \\
\hline $034512.5+321529$ & 17.87 \\
\hline $034512.5+320237$ & 17.89 \\
\hline $034512.6+321105$ & 19.11 \\
\hline $034512.6+320513$ & 20.25 \\
\hline $034512.6+321640$ & 20.04 \\
\hline $034512.6+320947$ & 15.49 \\
\hline $034512.6+321406$ & 19.34 \\
\hline $034512.7+321145$ & 18.18 \\
\hline $034512.7+321116$ & 15.79 \\
\hline $034512.7+321156$ & 20.17 \\
\hline $034512.8+320316$ & 14.54 \\
\hline $034512.8+320923$ & 17.50 \\
\hline $034512.8+321409$ & 19.69 \\
\hline $034512.9+320336$ & 18.03 \\
\hline $034512.9+321258$ & 18.31 \\
\hline $034513.1+320359$ & 20.17 \\
\hline $034513.1+320829$ & 19.66 \\
\hline $034513.2+321258$ & 18.89 \\
\hline $034513.2+321527$ & 14.15 \\
\hline $034513.4+320140$ & 18.97 \\
\hline $034513.4+321611$ & 19.71 \\
\hline $034513.4+321138$ & 19.58 \\
\hline $034513.5+320551$ & 18.56 \\
\hline $034513.5+320923$ & 17.74 \\
\hline $034513.5+321444$ & 19.35 \\
\hline $034513.6+320212$ & 16.02 \\
\hline $034513.6+321018$ & 19.29 \\
\hline $034513.6+320120$ & 11.60 \\
\hline $034513.6+321348$ & 16.07 \\
\hline $034513.7+320307$ & 19.40 \\
\hline $034513.8+321210$ & 16.85 \\
\hline $034513.8+321408$ & 18.12 \\
\hline $034513.9+320924$ & 18.76 \\
\hline $034513.9+321414$ & 19.03 \\
\hline
\end{tabular}

\begin{tabular}{|c|c|}
\hline IC348-J & $\begin{array}{c}J \\
{[\mathrm{mag}]}\end{array}$ \\
\hline $034514.0+320150$ & 16.02 \\
\hline $034514.0+321144$ & 15.06 \\
\hline $034514.0+320652$ & 20.29 \\
\hline $034514.0+321245$ & 19.91 \\
\hline $034514.0+321120$ & 16.92 \\
\hline $034514.1+320951$ & 18.88 \\
\hline $034514.1+321716$ & 19.11 \\
\hline $034514.2+320802$ & 20.22 \\
\hline $034514.3+321630$ & 14.33 \\
\hline $034514.3+320909$ & 20.45 \\
\hline $034514.4+321552$ & 14.62 \\
\hline $034514.4+320315$ & 20.04 \\
\hline $034514.4+320832$ & 18.23 \\
\hline $034514.4+321251$ & 19.34 \\
\hline $034514.5+320741$ & 19.56 \\
\hline $034514.7+321321$ & 20.05 \\
\hline $034514.8+320549$ & 17.08 \\
\hline $034514.8+321630$ & 18.99 \\
\hline $034514.8+321328$ & 17.66 \\
\hline $034514.8+321538$ & 19.02 \\
\hline $034514.9+321109$ & 17.56 \\
\hline $034515.0+321626$ & 17.30 \\
\hline $034515.1+320940$ & 19.46 \\
\hline $034515.1+321543$ & 19.71 \\
\hline $034515.1+321439$ & 20.06 \\
\hline $034515.2+321030$ & 18.90 \\
\hline $034515.2+321309$ & 19.56 \\
\hline $034515.2+321505$ & 20.01 \\
\hline $034515.3+321230$ & 15.83 \\
\hline $034515.4+321113$ & 17.09 \\
\hline $034515.4+321415$ & 17.49 \\
\hline $034515.6+320650$ & 19.83 \\
\hline $034515.7+320812$ & 11.32 \\
\hline $034515.7+321209$ & 13.80 \\
\hline $034515.7+320754$ & 19.56 \\
\hline $034515.8+321618$ & 19.70 \\
\hline $034515.8+320222$ & 19.23 \\
\hline $034515.8+321237$ & 16.59 \\
\hline $034515.8+320325$ & 16.38 \\
\hline $034515.9+320915$ & 20.05 \\
\hline $034516.0+321000$ & 19.64 \\
\hline $034516.0+321230$ & 19.49 \\
\hline $034516.0+320827$ & 19.78 \\
\hline $034516.0+321202$ & 20.22 \\
\hline $034516.1+320514$ & 19.52 \\
\hline $034516.1+321432$ & 20.07 \\
\hline $034516.1+320232$ & 16.01 \\
\hline $034516.1+321118$ & 18.29 \\
\hline $034516.2+321007$ & 20.00 \\
\hline $034516.2+321018$ & 17.27 \\
\hline $034516.2+321718$ & 16.22 \\
\hline $034516.2+320648$ & 19.73 \\
\hline $034516.3+320619$ & 11.97 \\
\hline $034516.4+321303$ & 18.78 \\
\hline $034516.4+321652$ & 14.89 \\
\hline $034516.4+321546$ & 18.06 \\
\hline $034516.5+320942$ & 15.13 \\
\hline
\end{tabular}

\begin{tabular}{|c|c|}
\hline IC348-J & $\begin{array}{c}J \\
{[\mathrm{mag}]}\end{array}$ \\
\hline $034516.6+321220$ & 15.19 \\
\hline $034516.7+321432$ & 19.44 \\
\hline $034516.8+320817$ & 19.79 \\
\hline $034516.8+321712$ & 20.11 \\
\hline $034516.9+321709$ & 19.62 \\
\hline $034516.9+320611$ & 16.15 \\
\hline $034516.9+320319$ & 17.47 \\
\hline $034516.9+321650$ & 20.17 \\
\hline $034517.0+321535$ & 18.71 \\
\hline $034517.0+320412$ & 19.84 \\
\hline $034517.0+321654$ & 18.21 \\
\hline $034517.1+320755$ & 17.40 \\
\hline $034517.2+321037$ & 20.15 \\
\hline $034517.3+320952$ & 19.91 \\
\hline $034517.3+321040$ & 20.00 \\
\hline $034517.3+321636$ & 18.24 \\
\hline $034517.4+321359$ & 13.33 \\
\hline $034517.4+321637$ & 19.25 \\
\hline $034517.4+321250$ & 17.32 \\
\hline $034517.5+321034$ & 16.09 \\
\hline $034517.5+320750$ & 20.19 \\
\hline $034517.6+320646$ & 19.87 \\
\hline $034517.6+321135$ & 20.11 \\
\hline $034517.6+320755$ & 18.32 \\
\hline $034517.7+320837$ & 18.83 \\
\hline $034517.8+321414$ & 20.08 \\
\hline $034517.8+320705$ & 19.93 \\
\hline $034517.8+320929$ & 18.99 \\
\hline $034517.8+321206$ & 13.75 \\
\hline $034517.9+321606$ & 20.56 \\
\hline $034517.9+321545$ & 17.41 \\
\hline $034517.9+321600$ & 18.89 \\
\hline $034518.0+321301$ & 18.67 \\
\hline $034518.0+321146$ & 20.25 \\
\hline $034518.1+321031$ & 19.95 \\
\hline $034518.1+321459$ & 20.44 \\
\hline $034518.1+321404$ & 16.54 \\
\hline $034518.2+320754$ & 17.82 \\
\hline $034518.2+320852$ & 16.36 \\
\hline $034518.2+321422$ & 14.89 \\
\hline $034518.2+320951$ & 18.97 \\
\hline $034518.2+320940$ & 17.76 \\
\hline $034518.2+320743$ & 17.45 \\
\hline $034518.3+320641$ & 17.06 \\
\hline $034518.3+320648$ & 19.34 \\
\hline $034518.3+321441$ & 18.91 \\
\hline $034518.4+320901$ & 19.24 \\
\hline $034518.4+321351$ & 19.91 \\
\hline $034518.4+320615$ & 19.20 \\
\hline $034518.5+321251$ & 18.23 \\
\hline $034518.6+320949$ & 19.57 \\
\hline $034518.7+320331$ & 20.21 \\
\hline $034518.7+321033$ & 19.99 \\
\hline $034518.8+321042$ & 19.25 \\
\hline $034518.8+321646$ & 14.60 \\
\hline $034518.9+320301$ & 20.26 \\
\hline $034519.2+320239$ & 18.69 \\
\hline
\end{tabular}

